Teste para avaliar a propriedade de incrementos independentes em um processo pontual. 

SERVIÇO DE PÓS-GRADUAÇÃO DO ICMC-USP

Data de Depósito:

Assinatura:

\title{
Teste para avaliar a propriedade de incrementos independetes em um processo pontual
}

\author{
Francys Andrews de Souza
}

Orientador: Prof. Dr. Dorival Leão Pinto Júnior

\begin{abstract}
Dissertação apresentada ao Instituto de Ciências Matemáticas e de Computação - ICMC-USP, como parte dos requisitos para obtenção do título de Mestre em Ciências - Ciências de Computação e Matemática Computacional. VERSÃO REVISADA
\end{abstract}


Ficha catalográfica elaborada pela Biblioteca Prof. Achille Bassi e Seção Técnica de Informática, ICMC/USP com os dados fornecidos pelo(a) autor(a)

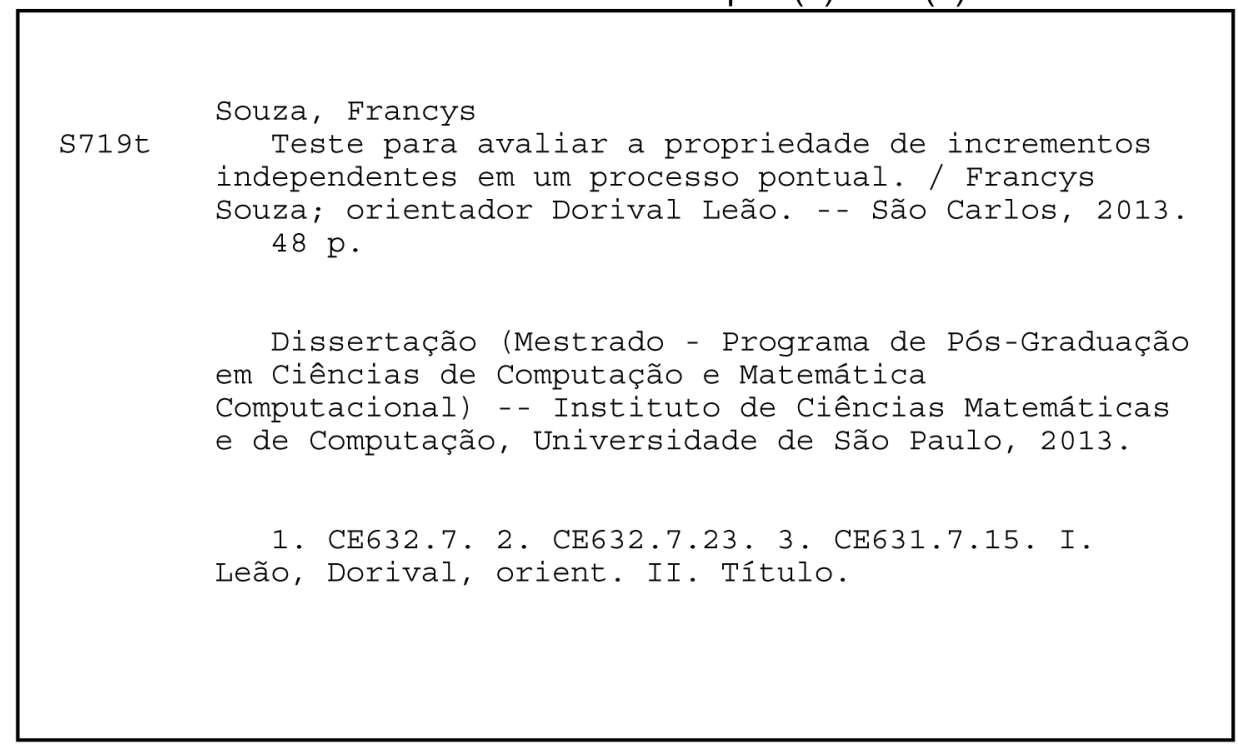




\section{Agradecimentos}

Agradeço a Deus por te me dado a oportunidade de desenvolver essa pesquisa e por ter me capacitado a concluí-la. Aos meus pais Alaor e Marta por todo o apoio e o esforço que fizeram para que eu pudesse chegar até aqui, me ajudando nos momentos difíceis e a minha irmã Patrícia por ter me apoiado, devo muito a eles. Agradeço a minha noiva Lidiane por todo auxílio sua compreensão sem limite durante o período que estive ausente para me dedicar ao desenvolvimento deste trabalho, companheirismo e por me dar suporte e a força necessária para que pudesse trilhar esse árduo caminho. Agradeço ao meu orientador Dorival Leão pela paciência, amizade, dedicação e por todo conhecimento que pude adquirir com sua experiência, além da confiança depositada em mim. Agradeço também carinhosamente aos meus amigos Bona, Cabeça, Hiro, Rafael e Rubens. Em especial sou grato aos meus grandes amigos e companheiros Lucas e Victor por toda ajuda e generosidade.

Finalmente, ao $\mathrm{CNPq}$ agradeço pelo apoio financeiro cedido. 

Em econometria um dos tópicos que vem se tornando ao longo dos anos primordial é a análise de ultra-frequência, ou seja, a análise da transação negócio a negócio. Ela tem se mostrado fundamental na modelagem da microestrutura do mercado intraday. Ainda assim temos uma teoria escassa que vem crescendo de forma humilde a cerca deste tema. Buscamos desenvolver um teste de hipótese para verificar se os dados de ultra-frequência apresentam incrementos independentes e estacionários, pois neste cenário saber disso é de grande importância, já que muitos trabalhos tem como base essa hipótese. Além disso Grimshaw et. al. (2005) 6] mostrou que ao utilizarmos uma distribuição de probabilidade contínua para modelarmos dados econômicos, em geral, estimamos uma função de intensidade crescente, devido a resultados viciados obtidos como consequência do arredondamento, em nosso trabalho buscamos trabalhar com distribuições discretas para que contornar esse problema acarretado pelo uso de distribuições contínuas. 

In econometrics a topic that is becoming primordial over the years is the ultra frequency analysis, or analysis of the trades to trades transaction. This topic is shown to be fundamental in modeling the microstructure of the market intraday. Nevertheless we have a little theory that is growing so lowly about this topic. We seek to develop a hypothesis test to verify that the data ultrasonic frequency have independent and stationary increments, for this scenario the knowledge of it great importance, since many jobs is based on this hypothesis. In general Grimshaw et. al. (2005) [6] showed that when we use a continuous probability distribution to model ecomomic data, we estimate a function of increasing intensity due to addicts results obtained as a result of rounding. In our research we seek to work with discrete distributions to circumvent this problem entailed by the use of continuous distributions. 

$\begin{array}{lll}\mathbf{1} & \text { Introdução } & \mathbf{1}\end{array}$

1.1 Descrição dos Dados $\ldots \ldots \ldots \ldots \ldots \ldots \ldots$

\begin{tabular}{|lll}
2 & Modelagem para função intensidade. & $\mathbf{7}$
\end{tabular}

2.1 Definição das variáveis $\ldots \ldots \ldots \ldots \ldots \ldots$. . . . . . . . . . . . . . . . . . . . . .

2.2 Decomposição de Doob-Meyer e o Compensador $\ldots \ldots \ldots \ldots$. . . . . . 8

$2.2 .1 \quad$ Compensador Processo pontual . . . . . . . . . . . . . . . 8

$2.2 .2 \quad$ Caso Simples . . . . . . . . . . . . . . . . . . . . 10

$2.2 .3 \quad$ Caso Geral $\ldots \ldots \ldots \ldots \ldots$

2.3 Medida Aleatória $\ldots \ldots \ldots \ldots$. . . . . . . . . . . . . . . . . 15

2.3 .1 Compensador Medida Aleatória . . . . . . . . . . . . . . . . . . . 16

2.4 Processos com incrementos e independentes e estacionários. . . . . . . . . . . 17

3 Distribuição assintótica da estatística de logrank. 23

3.1 Hipótese nula não especificada e a conjectura de Simes. . . . . . . . . . . . . 25

$3.2 \quad$ Estudo de Simulação $\ldots \ldots \ldots \ldots \ldots$. . . . . . . . . . . . . . 27

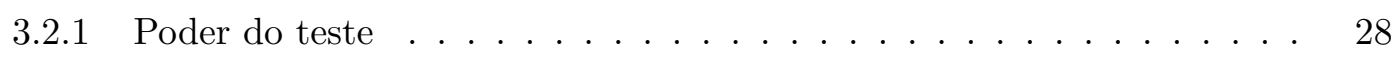

\begin{tabular}{lll}
\hline 4 & Teste exato. & 31
\end{tabular}

4.1 Distribuição exata do teste. . . . . . . . . . . . . . . . . . 33

\begin{tabular}{|lll}
\hline 5 & Teste para a PETR4 & 39
\end{tabular}

\begin{tabular}{ll}
\hline Conclusão & 45
\end{tabular}

\begin{tabular}{ll}
\hline Referências Bibliográficas & 45 \\
\hline
\end{tabular} 



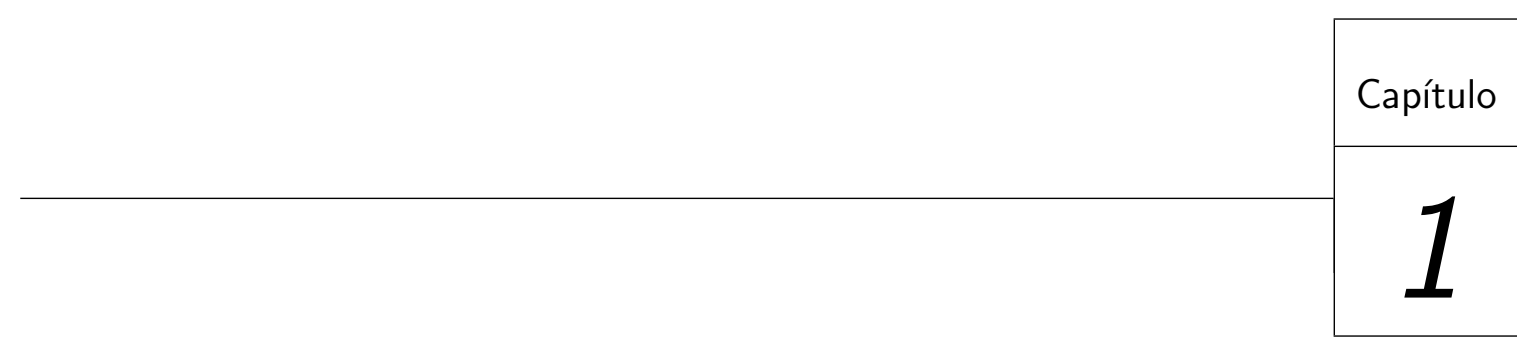

Introdução

Desde os trabalhos de Hasbrouck (1991)[7] e Engle and Russel (1998) [5] a modelagem de dados financeiros ao nível de transações (ou negócios) tem se tornado um tópico essencial da econometria, conhecido como análise de dados de ultra-frequência. As principais características dos dados de transações financeiras, como tempo irregular entre transações, a presença de forte sazonalidade dentro do dia (intraday) e erros de medição (exemplo: bid-ask bounce) levou ao desenvolvimento de modelos específicos para tratar estes dados. Para explorarmos as informações contidas nos dados das transações financeiras devemos utilizar modelos estatísticos que considerem o tempo entre ocorrência de transações de forma irregular. O tempo entre as transações e a frequência de transações carregam informações importantes sobre a microestrutura do mercado, que devem ser consideradas para modelar a volatilidade no dia (intraday), prever a liquidez do ativo e custo de transação.

Para considerarmos o tempo de ocorrência entre as transções vamos utilizar os modelos de processos pontuais financeiros. Neste caso, modelamos o tempo entre ocorrência do evento de interesse via processos pontuais com processo intensidade apropriados. No caso financeiro, o evento de interesse pode estar relacionado com o tempo de chegada de negócios ou o tempo de chegada de cotações, contabilizados a partir do momento de abertura da bolsa. Outro evento de interesse é a duração do preço do ativo, que corresponde ao tempo entre alterações do preço do mesmo. Para maiores detalhes e ilustrações, ver Bauwens e Giot (2001) [1] ou Hautsch (2004) [8].

Os modelos clássicos de taxa de falha aplicados na análise de sobrevivência, como o modelo de Cox ou modelo de testes acelerados, podem ser aplicados para modelar a intensidade 
de transações, ver Bauwens e Giot (2001)[1]. Entretanto, estes modelos são construídos com base na independência entre ocorrência de transações, fato que deve ser testado estatisticamente. Outra estratégia, consiste em utilizar os modelos de processos intensidade. Neste contexto, foram desenvolvidos extensões do processo de Poisson para permitir que a função intensidade seja aleatória e com isso, incluir uma estrutura de dependência entre os tempos entre ocorrência de transações, como o processo de Cox e o processo de Hawkes (ver, Hawkes (1971)[9]). Uma discussão detalhada sobre estas extensões do processo de Poisson pode ser encontrada em Rydberg e Shephard (2003)[15] e Heinen e Rengifo (2007)[10].

Uma forma alternativa consiste em modelarmos o processo intensidade através de um processo autoregressivo, o que nos levou a uma nova classe de processos pontuais denominados modelo de duração condicional autoregressivo (ACD). Um dos primeiros modelos de processo intensidade com duração condicional autoregressivo foi proposto por Engle e Russel (1998) [5]. A ideia essencial do modelo ACD é modelar o tempo entre ocorrência de transações em termos de um modelo de erro multiplicativo (ver, Engle (2002) [4]).

Uma particularidade da maioria dos trabalhos sobre processo pontual financeiro é que estes consideram o tempo de ocorrência das transações contínuo. Entretanto, devido a diversas limitações tecnológicas, os dados observados são sempre arredondados. Por exemplo, ao considerarmos o tempo de ocorrência de negócios das ações da Petrobras na BOVESPA, os dados são da escala de milesegundos. Grimshaw et. al. (2005) [6] mostrou que ao utilizarmos uma distribuição de probabilidade contínua para modelarmos dados econômicos, em geral, estimamos uma função de intensidade crescente, devido a resultados viciados obtidos como consequência do arredondamento. Para contornar o problema de arredondamento, Engle e Russel (1998) [5] descartaram as transações que ocorreram no mesmo instante, fato que gera vícios nas estimativas e na interpretação do modelo.

Outra alternativa, explorada por Höglund (2011) [12], consiste em truncar apropriadamente distribuições contínuas e tratar o tempo de ocorrência de negócios como dados intervalares. Neste contexto, Höglund (2011) [12] estudou a independência dos tempos entre ocorrência de transações ao longo dos dias e propôs a distribuição gama truncada para modelar estes tempos.

Analisamos os tempos de ocorrência de negócios das ações da Petrobras negociada na BOVESPA durante o mês de fevereiro de 2013. Observamos todas as transações ocorridas durante o dia registradas na escala de milesegundos tendo um total de $\left(27 \cdot 10^{6}\right.$ milesegundos $)$. Buscamos fazer um teste de hipótese para verificarmos se os dados possuem incrementos independentes e estacionários dentro do âmbito da ultra-frequência baseado no processo de pontos marcados a tempo discreto. A partir deste processo, definimos uma filtragem apropriada e obtemos explicitamente o processo intensidade (via a decomposição de Doob-Meyer). Para processos a tempo discreto, Yu et. al. (2008)[19] fizeram uma revisão dos principais modelos de processo intensidade e estabeleceram relações entre eles. 
A principal contribuição deste trabalho está relacionada à aplicação do modelo de pontos marcados a tempo discreto em um cenário de dados de ultra-frequência. Fizemos a decomposição de Doob-Meyer, com o compesador em mãos analisamos sob quais circuntância os dados possuem incrementos independentes e estacionários, e mostramos que para os nossos dados, assumir essa hipótese é um erro. Apesar de ser muito comum na literatura considerar essa hipótese (Bauwens e Giot (2001)[1] e Hawkes (1971)[9]). Além disso, fomos capaz de considerar negócios que ocorreram no mesmo instante, consideração esta que não foi feita no trabalho de Engle e Russel (2002) [4]. Desenvolvemos a distribuição exata do teste proposto utilizando o estimador de máxima verossimilhança (EMV). Além de aplicarmos nosso teste de hipótese fazendo um estudo de simulação e por fim aplicamos em dados reais, sendo estes os dados da PETR4.

\subsection{Descrição dos Dados}

Nosso trabalho tem o objetivo de modelar a intensidade de negócios das ações da Petrobrás (PETR4) e verificar se os dados apresentam incrementos independentes e estacionários. A escolha da PETR4 é devido ao alto índice de negociações dia deste papel na bolsa de valores de São Paulo (BOVESPA), sendo a empresa brasileira mais negociada.

A Petrobrás é uma companhia brasileira de petróleo, sendo uma empresa de capital aberto cujo acionista majoritário é o governo brasileiro. Está entre as cinco maiores empresas do mundo em valor de mercado, com valor aproximado de $\mathrm{R} \$ 360$ bilhões, sendo que no final do segundo trimestre de 2008, antes da crise, ela chegou a valer $\mathrm{R} \$ 457,4$ bilhões. Suas ações são divididas em dois tipos PETR4 e PETR3. A PETR3 são ações do tipo ordinárias, ou seja, ações cujo o acionista tem o direito de voto nas assembléias gerais. A PETR4 são ações do tipo preferenciais, sem direito a voto e com preferência na distribuição dos dividendos.

Os dados apresentados a seguir (figura 1.1) mostram o valor da ação negócio a negócio(em milisegundos) dos dias 14 ao dia 18 de fevereiro 2013. Nosso estudo foi realizado utilizando um das semana do mês de janeiro de 2013. Os gráficos apresentados também mostram o valor da ação negócio a negócio e suas variações em intervalos de milisegundos.

Estamos levando em consideração apenas os dados referentes ao pregão normal (10h as 17:30h), pois no chamado after-market existem restrições quanto ao valor e a quantidade de ações a ser negociada. Desta forma, optamos por desconsiderar esse período do mercado e considerarmos apenas o período de negociação normal da bolsa. Cada um dos dias do período estudado teve um alto indice de negociações, como podemos ver na tabela abaixo.

Abaixo podemos ver a contagem do número de negócios ocorrido em cada instante em que ocorreu no dia. Note que a contagem é acumulativa.

Note que na figura podemos observar que o número de negócios foi mais intenso no dia 14 de fevereiro, ou seja, na segunda feira. Já na quarta feira apresentou a menor quantidade de 


\begin{tabular}{cc}
\hline Dias & No de Negociações \\
\hline $14 / 01 / 2013$ & 33354 \\
$15 / 01 / 2013$ & 27188 \\
$16 / 01 / 2013$ & 15068 \\
$17 / 01 / 2013$ & 20106 \\
$18 / 01 / 2013$ & 17822 \\
Média & 28384.5 \\
\end{tabular}

Tabela 1.1: Tabela do número de negócios ocorrido em cada um dos dias do período estudado.
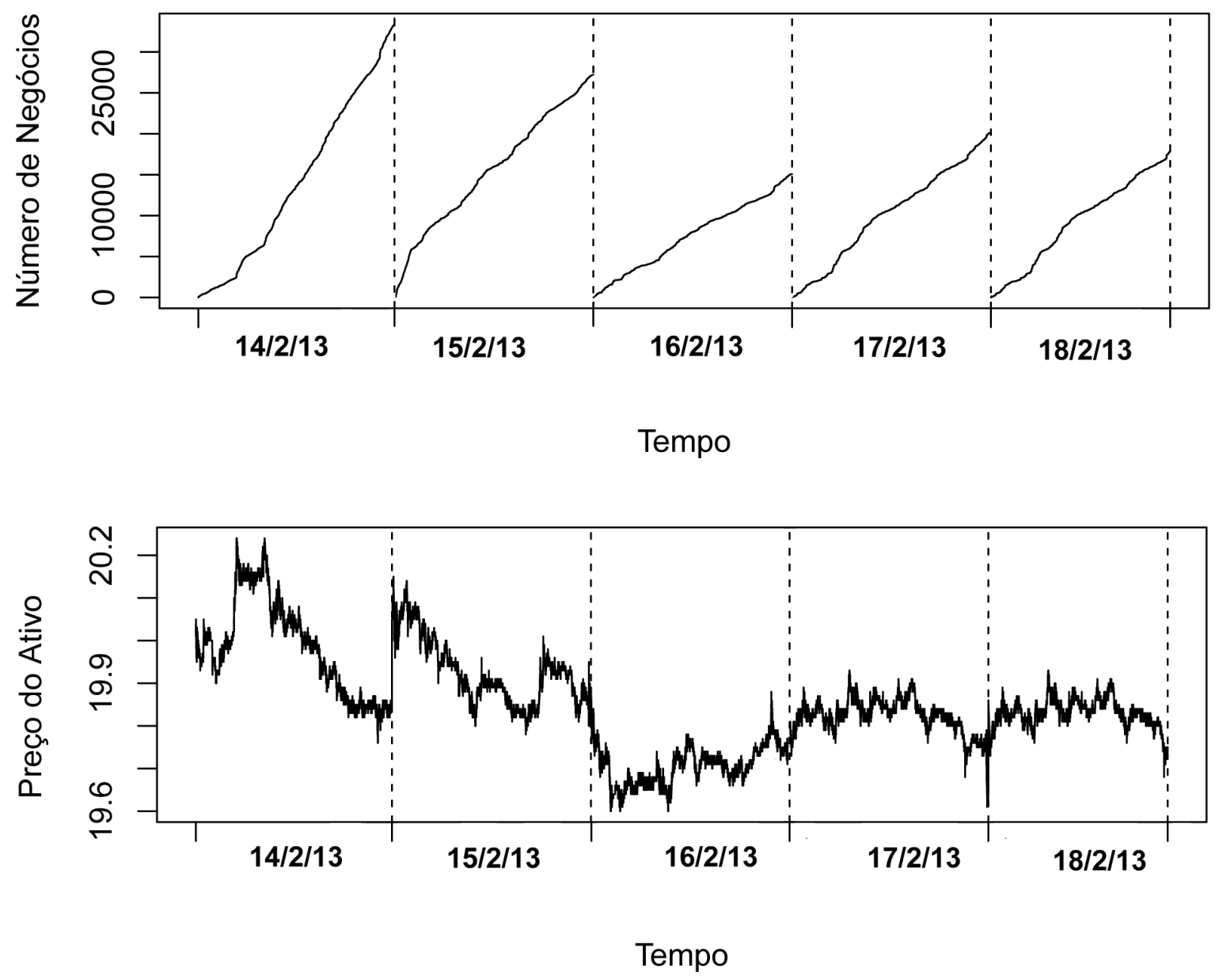

Figura 1.1: Número de negócios e preço da PETR4 do 14/02/2013 ao dia 18/02/2013.

negociações, note também que neste dia o ativo aprensentou o menor valor. Além disso podemos ver uma melhora no número de negociações na quinta e na sexta feira, além de uma sutil melhora no preço do ativo no mesmo período. Podemos notar também que na segunda feira aonde ocorreu o maior número das negociações da semana sendo quase o dobro do número de negociações da quarta feira, note que apresenta uma maior inclinação e consecutivamente 
uma maior intensidade no número de negociações justamente quando o preço do ativo começa a diminuir, na parte final do dia.

A dissertação está organizada da seguinte forma, no capitulo 2 modelamos o processo de contagem associado ao número de negócios e encontramos seu compensador. No capitulo 3, mostramos a distribuição assintótica da estatística de logrank e apresentamos um teste adequado ao modelo, teste este que verifica se os incrementos apresentam incrementos independentes e estacionários. No capitulo 4 apresentamos a distribuição exata do teste e por fim no capitulo 5 fizemos o teste de hipótese utilizando os dados da PETR4. 



$\frac{-10}{2}$

\section{Modelagem para função intensidade.}

Nesta seção apresentamos o nosso modelo utilizando um processo pontual, descrevemos nossas variável aleatórias. Definimos adequadamente nossa base estocástica $\left(\Omega, \mathcal{F},\left\{\mathcal{F}_{i}\right\}_{i \geq 1}, \mathbb{P}\right)$. Em seguida fizemos a decomposição de Doob-Meyer e analizamos, o que deve acontecer com o compensador para que nosso processo tenha incrementos independentes e $\backslash$ ou estacionários.

\subsection{Definição das variáveis}

Fixamos um espaço de probabilidade fundamental $(\Omega, \mathcal{F}, \mathbb{P})$, o qual utilizamos durante todo o nosso trabalho. Sejam $0=T_{0} \leq T_{1} \leq T_{2} \leq T_{3} \leq \cdots$ variáveis aleatórias discretas não negativas, sendo $T_{i}$ o tempo de ocorrência da i-ésima transação e $T$ o conjunto de todos os $T_{i}$.

O conjunto de dados utilizado tem os tempos medidos em milesegundos no período das dez da manhã até às cinco e meia da tarde totalizando um total de $27 \cdot 10^{6}$ milesegundos por dia. Para iniciar a modelagem, definiremos um processo pontual $N=\{N(\cdot, k): k \in \mathbb{N}\}$, no qual $\mathbb{N}$ representa o conjunto dos números naturais incluindo o zero, e $N(\cdot, k)$ é definido da seguinte forma: 


$$
\begin{aligned}
N(\cdot, k) & =\sum_{i=1}^{\infty} \mathbb{1}_{\left\{T_{i} \leq k\right\}} \\
& =\sum_{i=1}^{\infty} \sum_{\ell=1}^{k} \mathbb{1}_{\left\{T_{i}=\ell\right\}} \\
& =\sum_{\ell=1}^{k} \sum_{i=1}^{\infty} \mathbb{1}_{\left\{T_{i}=\ell\right\}}, \quad k=0,1, \ldots
\end{aligned}
$$

Note que o processo $N$ é um processo de contagem que conta o número de negócios ocorridos até o tempo $k$. Abaixo podemos ver um gráfico do comportamento do processo de contagem $N$ sobre os dados da PETR4 do dia 14 de fevereiro de 2013.

Neste momento, afim de levar em conta toda a informação gerada pela amostra, vamos criar uma filtragem $\left\{\mathcal{F}_{i}\right\}_{i \in \mathbb{N}}$, a qual é definida a seguir:

$$
\mathcal{F}_{i}=\sigma(N(\cdot, \ell): \ell \leq i, \forall \ell \in\{0,1, \cdots, i\}), \quad i \in \mathbb{N}
$$

Observe que o processo de contagem $N$ no zero pode ser diferente de zero, já que $P\left[T_{1}=\right.$ $0]>0$, assim podemos ter um número positivo de transações no instante zero. Decorrente deste fato vamos definir uma $\sigma$-álgebra inicial, Dellacherie e Meyer (pg 121) [3] trataram disto da mesma forma criando uma $\sigma$-algebra inicial, a qual chamaremos $\mathcal{F}_{-1}=\{\emptyset, \Omega\}$.

\subsection{Decomposição de Doob-Meyer e o Compensador}

A decomposição de Doob-Meyer é um dos mais importantes resultados da teoria de processos estocásticos. Esse teorema é fundamental, pois nos garante que podemos decompor um processo estocástico em uma parte previsível e um martingale. Um detalhe de grande importância deste teorema é que ele garante a unicidade da decomposição.

\subsubsection{Compensador Processo pontual}

O processo estocástico $N$ é um processo de contagem não decrescente e portanto um submartingale. Podemos obter a decomposição de Doob-Meyer para o processo de contagem $N$ já que ele é um processo discreto. Assim a partir da filtragem $\left\{\mathcal{F}_{i}\right\}$, obtemos a seguinte decomposição

$$
N(\cdot, \ell)=Y(\cdot, \ell)+\widetilde{N}(\cdot, \ell)
$$

no qual $Y(\cdot, \ell)=N(\cdot, \ell)-\widetilde{N}(\cdot, \ell)$ é a componente martingale da decomposição de DoobMeyer de $N$ com respeito à filtragem $\left\{\mathcal{F}_{i}\right\}_{i \in \mathbb{N}}$, a qual pode ser interpretada como um ruído. 
$14 / 02 / 2013$

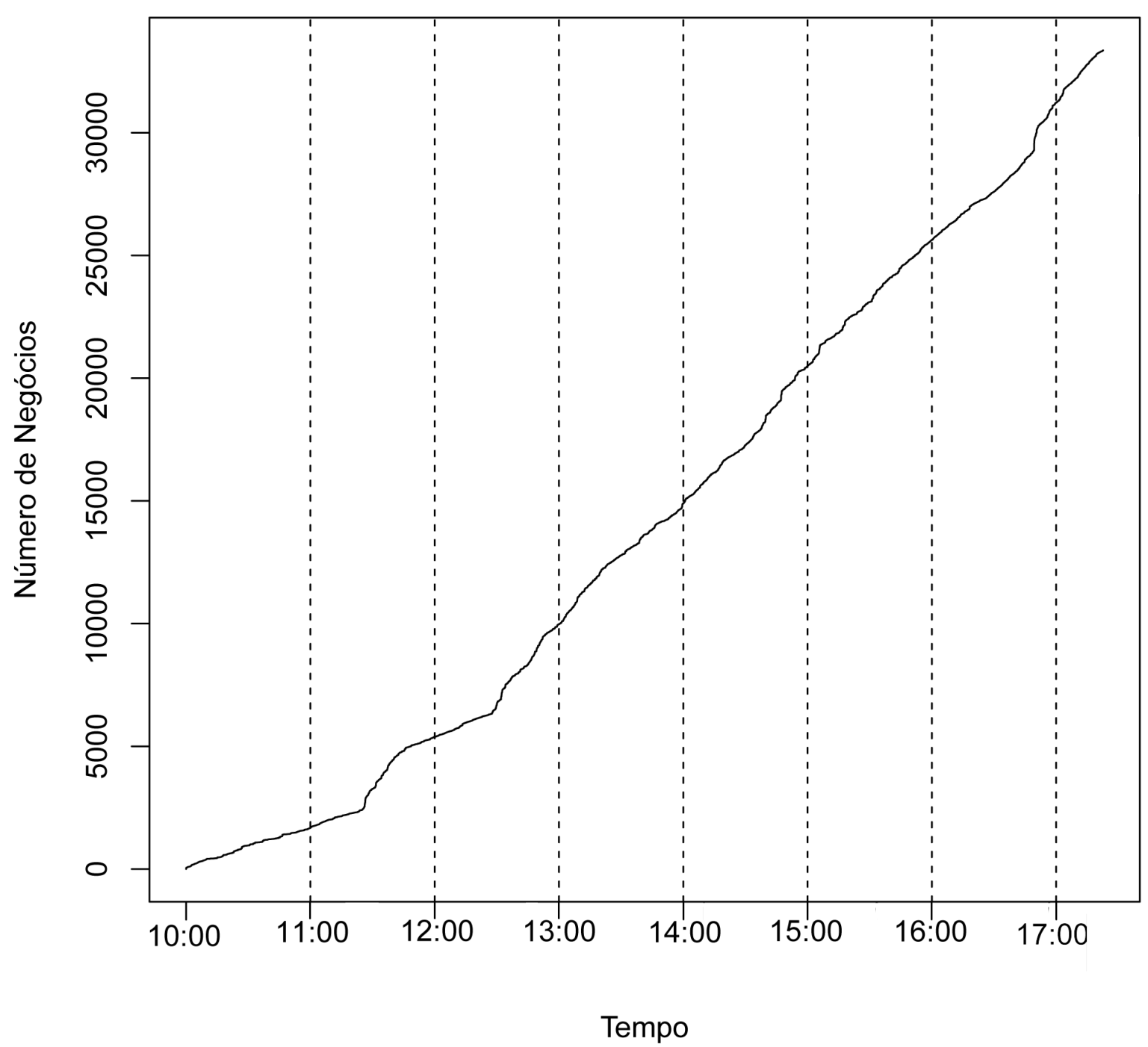

Figura 2.1: Processo de contagem $N$.

Temos portanto que $\widetilde{N}$ é a parte previsível da decomposição Doob-Meyer, também chamada de compensador. De acordo com Shiryayev (Teorema 2, pg 482) [17], a seguinte decomposição para o processo de contagem é válida

$$
N(\cdot, \ell)=Y(\cdot, \ell)+\sum_{j=1}^{\ell}\left[E\left(N(\cdot, j) \mid \mathcal{F}_{j-1}\right)-N(\cdot, j-1)\right] \quad \ell \geq 0 .
$$

Para mostrarmos a forma do nosso compensador vamos necessitar dividir em dois casos, o caso simples e o geral. No simples o conjunto de saltos do nosso processo de contagem 
contém apenas um elemento, já no caso geral nosso conjunto de saltos pode assumir um valor enumerável de valores.

\subsubsection{Caso Simples}

Neste caso utilizamos um processo de contagem simples $\rho(\cdot, \ell)$, com o conjunto $\mathcal{T}$ contendo apenas um elemento, denotado por $\tau$, o qual $\tau$ é a variável aleatória não negativa que representa o instante de ocorrência do primeiro negócio. Definimos o processo de contagem simples $\rho(\cdot, \ell)=\mathbb{1}_{\{\tau \leq \ell\}}$.

Dado $D$ uma variável aleatória, definimos por $\sigma(D)$ a menor $\sigma$-álgebra gerada pela variável $D$. Além disso seja $\tau$ uma variável aleatória não negativa e $\sigma$-álgebra $\mathcal{E}_{t}=\sigma(\min \{\tau, t\})$, com $t \in \mathbb{N}$, é importante observar que $\mathcal{E}_{t}=\sigma\left(\tau \mathbb{1}_{\tau \leq t}+t \mathbb{1}_{\tau>t}\right)$. Desta forma, podemos notar que $\sigma\left(\left\{\tau \mathbb{1}_{\tau \leq t}\right\}\right)=\sigma(\tau \cap\{\tau \leq t\})$. Observe também que, a nova variável gerada $\mathbb{1}_{\tau \leq t}+t \mathbb{1}_{\tau>t}$, tem como imagem inversa ao conjunto $(t, \infty)$ um átomo indivisível. Além disso definimos $\mathcal{E}_{-1}=\{\emptyset, \Omega\}$.

Para encontrarmos o compensador $A$ de $\rho$, vamos necessitar do teorema abaixo, cuja a demonstração pode ser encontrada no livro do Protter (Lema pg 119) (2004) 14].

Lema 2.2.1. Dado uma variável aleatória $W$ discreta, não negativa e integrável e $\tau$ uma variável aleatória não negativa. Seja $\mathcal{E}_{t}$ definida acima com $\mathcal{E}_{t}$ a menor filtragem que torna $\tau$ um tempo de parada. Portanto

$$
E\left[W \mid \mathcal{E}_{t}\right]=E[W \mid \tau] \mathbb{1}_{\{\tau \leq t\}}+\mathbb{1}_{\{\tau>t\}} \frac{E\left[\mathbb{1}_{\{\tau>t\}} W\right]}{P[\tau>t]},
$$

Demonstração: Por definição em $\tau$ a $\sigma$-álgebra $\mathcal{E}_{t}$ é igual a $\sigma$-álgebra das partes dos naturais até $t$, junto com átomo indivisível $B=\{t+1, t+2, \cdots\}$. Observe que $E\left[W \mid \mathcal{E}_{t}\right]$ é uma variável aleatória, assim temos que

$$
E\left[W \mid \mathcal{E}_{t}\right]=E\left[W \mid \mathcal{E}_{t}\right] \mathbb{1}_{\{\tau \leq t\}}+\mathbb{1}_{\{\tau>t\}} E\left[W \mid \mathcal{E}_{t}\right]
$$

Além disso,

$$
E\left[W \mid \mathcal{E}_{t}\right] \mathbb{1}_{\{\tau \leq t\}}=E[W \mid \tau] \mathbb{1}_{\{\tau \leq t\}},
$$

Além disso pelo fato de $B$ ser um átomo indivisível e pelo fato de $\left\{B, B^{c}\right\}$ formarem uma partição de $\mathbb{N}$, temos pela própria definição de esperança condicional que

$$
E\left[W \mid \mathcal{E}_{t}\right] \mathbb{1}_{\{\tau>t\}}=E[W \mid B] \mathbb{1}_{\{\tau>t\}}=\frac{E\left[\mathbb{1}_{\{\tau>t\}} W\right]}{P(\tau>t)} \mathbb{1}_{\{\tau>t\}}
$$

E portanto o resultado segue. 
Teorema 2.2.2. O compensador do processo de contagem $\rho(\cdot, \ell)$ é dado por $\sum_{j=0}^{\ell} \frac{P[\tau=j]}{P[\tau \geq j]} \mathbb{1}_{\{\tau \geq j\}}$, com $\ell \in \mathbb{N} \cup\{0\}$

Demonstração: A partir do lema2.2.1, definimos uma forma para o compensador. Utilizando a decomposição de Doob-Meyer segundo a filtragem $\mathcal{E}$ e o lema 2.2.1 temos que

$$
\begin{aligned}
E\left[\rho(\cdot, j) \mid \mathcal{E}_{j-1}\right] & =\rho(\cdot, j) \mathbb{1}_{\{\tau \leq j-1\}}+\frac{E\left[\mathbb{1}_{\{\tau>j-1\}} \rho(\cdot, j)\right]}{P[\tau>j-1]} \mathbb{1}_{\{\tau>j-1\}} \\
& =\mathbb{1}_{\{\tau \leq j-1\}}+\frac{E\left[\mathbb{1}_{\{j-1<\tau \leq j\}}\right]}{P[\tau>j-1]} \mathbb{1}_{\{\tau>j-1\}} \\
& =\mathbb{1}_{\{\tau \leq j-1\}}+\frac{P[\tau=j]}{P[\tau>j-1]} \mathbb{1}_{\{\tau>j-1\}} \\
& =\mathbb{1}_{\{\tau \leq j-1\}}+\frac{P[\tau=j]}{P[\tau>j-1]} \mathbb{1}_{\{\tau>j-1\}}, \quad j \in \mathbb{N} .
\end{aligned}
$$

Portanto nosso compensador é dado por

$$
\begin{aligned}
A(\cdot, \ell) & =\sum_{j=1}^{\ell}\left[E\left(\rho(\cdot, j) \mid \mathcal{E}_{j-1}\right)-\rho(\cdot, j-1)\right] \\
& =\sum_{j=1}^{\ell} \mathbb{1}_{\{\tau \leq j-1\}}+\frac{P[\tau=j]}{P[\tau>j-1]} \mathbb{1}_{\{\tau>j-1\}}-\mathbb{1}_{\{\tau \leq j-1\}} \\
& =\sum_{j=1}^{\ell} \frac{P[\tau=j]}{P[\tau>j-1]} \mathbb{1}_{\{\tau>j-1\}} \\
& =\sum_{j=1}^{\ell} \frac{P[\tau=j]}{P[\tau \geq j]} \mathbb{1}_{\{\tau \geq j\}}
\end{aligned}
$$

Podemos definir $h(j)=\frac{P[\tau=j]}{P[\tau \geq j]}$ e $V(j)=\mathbb{1}_{\{\tau \geq j\}}$ assim temos que nosso compensador é dado por $\sum_{j=1}^{\ell} \frac{P[\tau=j]}{P[\tau \geq j]} \mathbb{1}_{\{\tau \geq j\}}=\sum_{j=1}^{\ell} h(j) V(j)$

A função $h$ definida acima é conhecida como função intensidade e é de uso comum na literatura, principalmente na area de análise de sobrevivência. Sua definição é mais comum para o caso em que a variável aleatória $\tau$ é contínua. Como iremos trabalhar apenas com o caso puramente discreto vamos nos ater em utilizar apenas a definição para o caso em que a variável aleatória é discreta, o qual é dada da seguinte forma:

$$
h(j):=\frac{P[\tau=j]}{P[\tau \geq j]}, \quad j \in \mathbb{N},
$$


no qual $\tau$ é uma variável aleatória discreta, notem que podemos obter de forma única a distribuição de probabilidade da variável $\tau$ a partir da função intensidade $h$, definida da seguinte forma:

$$
P(\tau=j)=h(j) \prod_{\ell=1}^{j-1}[1-h(\ell)] .
$$

Esse resultado é demonstrado usando indução finita,

$$
\begin{gathered}
h(0)=P(\tau=0) \\
h(1)=\frac{P(\tau=1)}{P(\tau \geq 1)}=\frac{P(\tau=1)}{1-P(\tau=0)} \Rightarrow \\
P(\tau=1)=h(1)(1-h(0)) .
\end{gathered}
$$

Agora por indução em $j$ obtemos que:

$$
\begin{aligned}
& P(\tau=j)=h(j) \cdot P(\tau \geq j)=h(j)\left(1-\sum_{l=1}^{j-1} P(\tau=l)\right) \\
& =h(j)\left[1-\sum_{i=1}^{j} h(i) \prod_{l=1}^{i-2}(1-h(l))\right]=h(j) \prod_{l=1}^{j-1}[1-h(l)]
\end{aligned}
$$

Portanto essa relação é biunívoca, desta forma dado a função $h$ podemos encontrar a função de probabilidade da nossa variável aletória de forma única, da mesma forma dada a função de probabilidade podemos encontar a função $h$ de forma única.

Exemplo 2.2.3. Suponha que $\tau \sim G e o(\lambda)$, então sua função de probabilidade é dada por $P(\tau=j)=(1-\lambda)^{j-1} \lambda$. Sendo assim pela definição da função $h$ temos que:

$$
h(k)=\frac{P(\tau=k)}{P(\tau \geq k)}=\frac{(1-\lambda)^{k-1} \lambda}{1-\lambda \sum_{l=1}^{k-1}(1-\lambda)^{l}}=\frac{(1-\lambda)^{k} \lambda}{1-\frac{(1-\lambda)^{k+1}}{\lambda}}=\lambda
$$

Com o compensador do caso simples em mãos podemos encontrar o compensador do processo pontual no caso geral.

\subsubsection{Caso Geral}

Aqui tomamos o processo pontual original $N(\cdot, \ell)=\sum_{i=1}^{\infty} \mathbb{1}_{\left\{T_{i} \leq \ell\right\}}$, com $\ell \in \mathbb{N}$. Para encontrarmos o compensador de $N(\cdot, \ell)$, definimos primeiramente uma função $\varphi_{i}$, a qual é dada abaixo para : 


$$
\begin{aligned}
\varphi_{1}(\cdot, t) & =\sum_{\ell=0}^{t} \frac{P\left[T_{1}=\ell \mid \mathcal{F}_{0}\right]}{P\left[T_{1} \geq \ell \mid \mathcal{F}_{0}\right]}, \quad t \in\left(0 ; T_{1}\right] \\
\varphi_{i}(\cdot, t) & =\sum_{\ell=0}^{t-T_{i-1}} \frac{P\left[D_{i}=\ell \mid \mathcal{F}_{i-1}\right]}{P\left[D_{i} \geq \ell \mid \mathcal{F}_{i-1}\right]}, \quad t \in\left(T_{i-1} ; T_{i}\right]
\end{aligned}
$$

no qual $D_{i}$ é a variável aleatória definida como sendo $D_{i}=T_{i}-T_{i-1}$, que representa o tempo entre negócios. Utilizando uma abordagem diferente da empregada para $\rho(\cdot, \ell)$, usaremos diretamente a definição do compensador. Segue da unicidade da decomposição de Doob, que se $D=\left(D_{i}\right)_{i \in \mathbb{N}}$ é um submartingale, então o seu compensador $C$ é o único processo previsível que satisfaz a seguinte propriedade: $D-C$ é um martingale.

Observação 2.2.4. É importante notar que quando $t \in\left(T_{i-1}, T_{i}\right]$ temos que $T_{i-1}<T_{i}$, pois caso $T_{i-1}=T_{i}$ então o conjunto $\left(T_{i-1}, T_{i}\right]=\emptyset$ e portanto $t=\emptyset$. Muitos autores buscaram eliminar os pontos em que $T_{i}-T_{i-1}=0$ como, por exemplo [5]. Entretanto em nosso caso não eleminamos esses pontos, apenas exigimos que os calculos em relação ao compensador sejam feitos para todo $t$ de forma que $\left(T_{i-1}, T_{i}\right] \neq \emptyset$. Desta forma, para cada $t \in \mathbb{N}$, tomamos o intervalo $\left(T_{i-1}, T_{i}\right]$, tal que $t \in\left(T_{i-1}, T_{i}\right]$, claro que $t$ deve ser menor ou igual ao $\max _{i \in \mathbb{N}}\left\{T_{i}\right\}$

Teorema 2.2.5. O processo de contagem $N$, para $t \in\left(T_{j-1}, T_{j}\right]$ tem compensador dado por:

$$
\begin{aligned}
\widetilde{N}(\cdot, t) & =\varphi_{1}\left(\cdot, D_{1}\right)+\varphi_{2}\left(\cdot, D_{2}\right)+\cdots+\varphi_{j-1}\left(\cdot, D_{j-1}\right)+\varphi_{j}\left(\cdot, t-T_{j-1}\right) \\
& =\sum_{\ell=1}^{\infty}\left\{\sum_{i=1}^{\ell-1}\left[\varphi_{i}\left(\cdot, D_{i}\right)+\varphi_{\ell}\left(\cdot, t-T_{\ell}\right)\right] \mathbb{1}_{\left\{T_{\ell-1}<t \leq T_{\ell}\right\}}\right\}, \quad t \in \mathbb{N} .
\end{aligned}
$$

Demonstração: Mostremos então que $Y(\cdot, t)=N(\cdot, t)-\widetilde{N}(\cdot, t)$ é um martingale. Seja $t \in\left(T_{j-1} ; T_{j}\right]$ então

$$
\begin{aligned}
Y(\cdot, t) & =N(\cdot, t)-\tilde{N}(\cdot, t) \\
& =\left(\sum_{i=1}^{\infty} \mathbb{1}_{\left\{T_{i} \leq t\right\}}\right) \mathbb{1}_{\left\{T_{j-1}<t \leq T_{j}\right\}}-\varphi_{1}\left(\cdot, D_{1}\right)-\varphi_{2}\left(\cdot, D_{2}\right)-\cdots-\varphi_{j}\left(\cdot, t-D_{j}\right) \\
& =\sum_{i=1}^{j} \mathbb{1}_{\left\{T_{i} \leq t\right\}}-\varphi_{1}\left(\cdot, D_{1}\right)-\varphi_{2}\left(\cdot, D_{2}\right)-\cdots-\varphi_{j}\left(\cdot, t-T_{j}\right) .
\end{aligned}
$$


Portanto,

$$
\begin{aligned}
E\left[Y(\cdot, t) \mid \mathcal{F}_{j-1}\right] & =\sum_{i=1}^{j-1} \mathbb{1}_{\left\{T_{i} \leq t\right\}} \\
& -\varphi_{1}\left(\cdot, D_{1}\right)-\varphi_{2}\left(\cdot, D_{2}\right)-\cdots-\varphi_{j-1}\left(\cdot, D_{j-1}\right) \\
& +E\left[\mathbb{1}_{\left\{T_{j} \leq t\right\}}-\varphi_{j}\left(\cdot, t-T_{j}\right) \mid \mathcal{F}_{j-1}\right] .
\end{aligned}
$$

Assim, para mostrarmos que $Y(\cdot, t)$ é um martingale, basta mostrarmos que $E\left[\mathbb{1}_{\left\{T_{j} \leq t\right\}}-\right.$ $\left.\varphi_{j}\left(\cdot, t-T_{j}\right) \mid \mathcal{F}_{j-1}\right]=0$. De fato,

$$
\begin{aligned}
E\left[\mathbb{1}_{\left\{T_{j} \leq t\right\}}-\varphi_{j}\left(\cdot, t-T_{j}\right) \mid \mathcal{F}_{j-1}\right] & =E\left[\mathbb{1}_{\left\{T_{j} \leq t\right\}} \mid \mathcal{F}_{j-1}\right]-E\left[\varphi_{j}\left(\cdot, t-T_{j}\right) \mid \mathcal{F}_{j-1}\right] \\
& =E\left[\mathbb{1}_{\left\{T_{j} \leq t\right\}} \mid \mathcal{F}_{j-1}\right]-\varphi_{j}\left(\cdot, t-T_{j}\right) \\
& =E\left[\mathbb{1}_{\left\{T_{j} \leq t\right\}} \mid \mathcal{F}_{j-1}\right] \\
& -\sum_{\ell=1}^{t-T_{j-1}} \frac{P\left[D_{j}=\ell \mid \mathcal{F}_{j-1}\right]}{P\left[D_{j} \geq \ell \mid \mathcal{F}_{j-1}\right]} \\
& =0
\end{aligned}
$$

pois

$$
\begin{aligned}
E\left[\mathbb{1}_{\left\{T_{j} \leq t\right\}} \mid \mathcal{F}_{j-1}\right] & =E\left[\mathbb{1}_{\left\{T_{j-1}+D_{j} \leq t\right\}} \mid \mathcal{F}_{j-1}\right] \\
& =E\left[\mathbb{1}_{\left\{D_{j} \leq t-T_{j-1}\right\}} \mid \mathcal{F}_{j-1}\right] \\
& =\sum_{\ell=1}^{t-T_{j-1}} E\left[\mathbb{1}_{\left\{D_{j}=\ell-T_{j-1}\right\}} \mid \mathcal{F}_{j-1}\right] \\
& =\sum_{\ell=1}^{t-T_{j-1}} \frac{P\left[D_{j}=\ell \mid \mathcal{F}_{j-1}\right]}{P\left[D_{j} \geq \ell \mid \mathcal{F}_{j-1}\right]}
\end{aligned}
$$

onde a última igualdade decorre da equação 2.3 , e portanto, temos que $Y(\cdot, t)$ é um martingale. Logo, concluímos que para $t \in\left(T_{j-1}, T_{j}\right]$ o nosso compensador é dado por:

$$
\begin{aligned}
\tilde{N}(\cdot, t) & =\varphi_{1}\left(\cdot, D_{1}\right)+\varphi_{2}\left(\cdot, D_{2}\right)+\cdots+\varphi_{j}\left(\cdot, t-T_{j}\right) \\
& =\sum_{\ell=1}^{\infty}\left\{\sum_{i=1}^{\ell-1}\left[\varphi_{i}\left(\cdot, D_{i}\right)+\varphi_{\ell}\left(\cdot, t-T_{\ell}\right)\right] \mathbb{1}_{\left\{T_{\ell-1}<t \leq T_{\ell}\right\}}\right\}, \quad t \in \mathbb{N} .
\end{aligned}
$$

Se adicionarmos a hipótese de que os $D_{i} \stackrel{\text { iid }}{\sim} F$ então temos que o compensador para $t \in\left(T_{j-1}, T_{j}\right]$ será dado por 


$$
\varphi_{i}(t)=\sum_{\ell=0}^{t-T_{j-1}} \frac{P\left(D_{1}=\ell\right)}{P\left(D_{1} \geq \ell\right)} .
$$

portanto, temos

$$
\widetilde{N}(\cdot, t)=\sum_{\ell=0}^{D_{1}} \frac{P\left(D_{1}=\ell\right)}{P\left(D_{1} \geq \ell\right)}+\cdots+\sum_{\ell=0}^{t-T_{j}} \frac{P\left(D_{1}=\ell\right)}{P\left(D_{1} \geq \ell\right)}
$$

Se supormos que a hipótese de que $N$ é um processo markoviano teremos que

$$
\begin{aligned}
\varphi_{1}(t) & =\sum_{\ell=0}^{t} \frac{P\left[D_{1}=\ell \mid N(\cdot, 0)\right]}{P\left[D_{1} \geq \ell \mid N(\cdot, 0)\right]} \\
\varphi_{i}(t) & =\sum_{\ell=0}^{t-T_{i-1}} \frac{P\left[D_{i}=\ell \mid N(\cdot, i-1)\right]}{P\left[D_{i} \geq \ell \mid N(\cdot, i-1)\right]}
\end{aligned}
$$

Nos capitulos seguintes usaremos a função intensidade abundantemente.

\subsection{Medida Aleatória}

Definimos um subconjunto $\{\mathcal{T}\}$ do conjunto $T$ de tal forma que cada $\tau_{i} \in \mathcal{T}$ é definido da seguinte forma:

$$
\begin{gathered}
\tau_{0}=0 \\
\tau_{1}=\inf \{\ell>0: \Delta N(\ell)>0\} \\
\vdots \\
\tau_{i}=\inf \left\{\ell>\tau_{i-1}: \Delta N(\ell)>0\right\}
\end{gathered}
$$

com $\Delta N(\ell)=N(\ell)-N(\ell-1)$ notemos que $\mathcal{T}$ contém apenas um de cada um dos $T_{i}$ 's distintos. Denotamos o processo $Z=\left\{Z_{\tau_{k}}: k \in \mathbb{N}\right\}$, tal que $Z_{\tau_{i}}$ define o número de negócios ocorridos no i-ésimo termo de $\mathcal{T}$, onde $Z_{\tau_{i}}$ é dado da seguinte forma:

$$
Z_{\tau_{i}}=\sum_{n=1}^{\infty} \mathbb{1}_{\left\{T_{n}=\tau_{i}\right\}}
$$

Assim, podemos reescrever o nosso processo de contagem $N$ utilizando a nova variável $Z_{\tau_{i}}$, o qual pode ser representado abaixo: 


$$
N(\cdot, k)=\sum_{i=1}^{\infty} Z_{\tau_{i}} \mathbb{1}_{\left\{\tau_{i} \leq k\right\}} .
$$

Desta forma, defina a medida aleatória $\mu: \Omega \times \mathbb{N} \times \mathbb{N} \rightarrow \mathbb{N}$, a medida é obtida da seguinte forma:

$$
\mu(\cdot, k, \ell)=\sum_{i=1}^{\infty} \mathbb{1}_{\left\{Z_{\tau_{i}}=k\right\}} \mathbb{1}_{\left\{\tau_{i} \leq \ell\right\}} .
$$

Então, temos que

$$
\begin{aligned}
N(\cdot, \ell) & =\sum_{k=1}^{\infty} k \mu(\cdot, k, \ell) \\
& =\sum_{k=1}^{\infty} k \sum_{i=1}^{\infty} \mathbb{1}_{\left\{Z_{\tau_{i}=k}\right\}} \mathbb{1}_{\left\{\tau_{i} \leq \ell\right\}} \\
& =\sum_{i=1}^{\infty} \mathbb{1}_{\left\{\tau_{i} \leq \ell\right\}} \sum_{k=1}^{\infty} k \mathbb{1}_{\left\{Z_{\tau_{i}=k}\right\}} \\
& =\sum_{i=1}^{\infty} Z_{\tau_{i}} \mathbb{1}_{\left\{\tau_{i} \leq \ell\right\}} .
\end{aligned}
$$

Observemos que esta medida aleatória é uma medida de contagem.

\subsubsection{Compensador Medida Aleatória}

Para todo $k \in \mathbb{N}$, o processo estocástico $\mu$ é um submartingale discreto. Então podemos obter a decomposição de Doob-Meyer para a medida aleatória $\mu$. A partir da filtragem $\left\{\mathcal{F}_{i}\right\}$, obtemos a seguinte decomposição

$$
\mu(\cdot, k, \ell)=M(\cdot, k, \ell)+r(\cdot, k, \ell)
$$

no qual $M(\cdot, k, \ell)=\mu(\cdot, \ell, k)-r(\cdot, k, \ell)$ é a componente martingale da decomposição de Doob-Meyer de $\mu$ com respeito à filtragem $\left\{\mathcal{F}_{i}\right\}$. Temos portanto que $r$ é a parte previsível da decomposição Doob-Meyer.

Seguindo os mesmos argumentos utilizados para encontrar o compensador do processo pontual temos que o compensador da medida aleatória para $t \in\left[\tau_{i-1}, \tau_{i}\right)$ é dado por

$$
\begin{gathered}
\phi_{1}(\cdot, k, t)=\sum_{\ell=1}^{t} \frac{P\left[\tau_{1}=\ell ; Z_{\tau_{1}}=k \mid \mathcal{F}_{0}\right]}{P\left[\tau_{1} \geq \ell \mid \mathcal{F}_{0}\right]} \\
\phi_{i}(\cdot, k, t)=\sum_{\ell=1}^{t-\tau_{i-1}} \frac{P\left[S_{i}=\ell ; Z_{\tau_{i}}=k \mid \mathcal{F}_{i-1}\right]}{P\left[S_{i} \geq \ell \mid \mathcal{F}_{i-1}\right]}
\end{gathered}
$$


$\operatorname{com} S_{i}=\tau_{i}-\tau_{i}$

$$
\begin{aligned}
r(\cdot, k, t) & =\phi_{1}\left(\cdot, k, \tau_{1}\right)+\phi_{2}\left(\cdot, k, S_{2}\right)+\cdots+\phi_{j}\left(\cdot, k, t-\tau_{j}\right)= \\
& =\sum_{i=1}^{\infty}\left\{\sum_{j=1}^{i-1}\left[\phi_{j}\left(\cdot, k, S_{j}\right)+\phi_{i}\left(\cdot, k, t-\tau_{i}\right)\right] \mathbb{1}_{\left\{\tau_{i-1}<t \leq \tau_{i}\right\}}\right\}
\end{aligned}
$$

\subsection{Processos com incrementos e independentes e estacionários.}

Sempre que nos propomos a fazer uma modelagem ponderamos algumas considerações e simplificações. Uma simplificação comum é a hipótese de que o processo de contagem $N$ tenha incrementos independentes.

Definição 2.4.1. Um processo estocástico $\left\{X_{t}, t \in \mathbb{N}\right\}$ discreto é dito ter incrementos independentes se para todo $0 \leq t_{0}<t_{1}$ a variável aleatória $\left(X_{t_{1}}-X_{t_{0}}\right)$ é independente da $\sigma$-álgebra $\mathcal{F}_{t_{0}}$

Lema 2.4.2. Para $u \in \mathbb{R}$ fixo e $0 \leq s \leq t$, o processo estocástico $Y_{t}=e^{i u(N(\cdot, t)-N(\cdot, s))}$ é um semimatingale com representação

$$
Y_{t}=1+\sum_{j=s+1}^{t} \sum_{k=0}^{\infty}\left[e^{i u k}-1\right] Y_{j-1} \Delta M(\cdot, k, j)+\sum_{j=s+1}^{t} \sum_{k=0}^{\infty}\left[e^{i u k}-1\right] Y_{j-1} \Delta r(\cdot, k, j)
$$

Demonstração: Para qualquer função $F: \mathbb{R} \rightarrow \mathbb{R}$, temos que

$$
\begin{aligned}
F(N(\cdot, t) & -N(\cdot, s))=F[0]+\sum_{j=s+1}^{t}(F[N(\cdot, j)-N(\cdot, s)]-F[N(\cdot, j-1)-N(\cdot, s)]) \\
& =F[0]+\sum_{j=s+1}^{t}(F[N(\cdot, j-1)+\Delta N(\cdot, j)-N(\cdot, s)]-F[N(\cdot, j-1)-N(\cdot, s)]) \\
& =F[0]+\sum_{j=s+1}^{t} \sum_{k=1}^{\infty}(F[N(\cdot, j-1)+k-N(\cdot, s)]-F[N(\cdot, j-1)-N(\cdot, s)]) \Delta \mu(\cdot, k, j)
\end{aligned}
$$

$\operatorname{com} \Delta r(\cdot, k, j)=r(\cdot, k, j)-r(\cdot, k, j-1)$ e $\Delta \mu(\cdot, k, j)=\mu(\cdot, k, j)-\mu(\cdot, k, j-1)$ Se tomarmos $F(x)=e^{i u x}$ e $Y_{t}=F(N(\cdot, t)-N(\cdot, s))$, concluímos que

$$
Y_{t}=1+\sum_{j=s+1}^{t} \sum_{k=0}^{\infty}\left[e^{i u k}-1\right] Y_{j-1} \Delta M(\cdot, k, j)+\sum_{j=s+1}^{t} \sum_{k=0}^{\infty}\left[e^{i u k}-1\right] Y_{j-1} \Delta r(\cdot, k, j)
$$

Teorema 2.4.3. O processo estocástico $N$ apresenta incrementos independentes se, e somente se, $\widetilde{N}$ é determinístico. 
Demonstração: Suponha $\tilde{N}(\cdot, t)=g(t)$, onde $g: \mathbb{N} \rightarrow \mathbb{R}$ é uma função real não aleatória. Desta forma, sabemos que o compensador da medida aleatória dado por $r$ também é determinístico. Para mostrarmos que $\mathrm{N}$ tem incrementos independentes, basta mostrarmos que

$$
E\left[e^{i u(N(\cdot, t)-N(\cdot, s))} \mid \mathcal{F}_{s}\right]=E\left[e^{i u(N(\cdot, t)-N(\cdot, s))}\right], \quad u \in \mathbb{R}
$$

ou de forma análoga para todo $A \in \mathcal{F}_{s}$

$$
E\left[\mathbb{1}_{A} e^{i u(N(\cdot, t)-N(\cdot, s))}\right]=P(A) E\left[e^{i u(N(\cdot, t)-N(\cdot, s))}\right]
$$

Ao denotarmos $Y_{t}=e^{i u(N(\cdot, t)-N(\cdot, s))}, \quad \forall t \geq s$ e $t \in \mathbb{N}$, obtemos do lema 2.4 .2 temos que $Y_{t}$ tem a seguinte representação

$$
Y_{t}=1+\sum_{j=s+1}^{t} \sum_{k=0}^{\infty}\left[e^{i u k}-1\right] Y_{j-1} \Delta M(\cdot, k, j)+\sum_{j=s+1}^{t} \sum_{k=0}^{\infty}\left[e^{i u k}-1\right] Y_{j-1} \Delta r(\cdot, k, j) .
$$

Além disso, como a parte martingale da decomposição de $Y_{t}$, é nula no ponto $t=s$, obtemos que

$$
E\left[\mathbb{1}_{A} \sum_{j=s+1}^{t} \sum_{k=0}^{\infty}\left[e^{i u k}-1\right] Y_{j-1} \Delta M(\cdot, k, j)\right]=0
$$

Desde que o compensador da medida aleatória é determinístico, concluímos que

$$
\begin{aligned}
E\left[\mathbb{1}_{A} Y_{t}\right] & =E\left[\mathbb{1}_{A}\right]+E\left[\mathbb{1}_{A} \sum_{j=s+1}^{t} \sum_{k=0}^{\infty}\left[e^{i u k}-1\right] Y_{j-1} \Delta r(\cdot, k, j)\right] \\
& =P(A)+\sum_{j=s+1}^{t} \sum_{k=0}^{\infty} E\left[\mathbb{1}_{A} Y_{j-1}\right]\left[e^{i u k}-1\right] \Delta r(\cdot, k, j) .
\end{aligned}
$$

Ao tomarmos $f_{t}=\frac{E\left[\mathbb{1}_{A} Y_{t}\right]}{P(A)}$, obtemos que 


$$
\begin{aligned}
f_{t} & =1+\sum_{j=s+1}^{t} \sum_{k=0}^{\infty} f_{j-1}\left[e^{i u k}-1\right] \Delta r(\cdot, k, j) \\
& =1+\sum_{j=s+1}^{t} f_{j-1} \sum_{k=0}^{\infty}\left[e^{i u k}-1\right] \Delta r(\cdot, k, j) \\
& =1+\sum_{j=s+1}^{t} f_{j-1} \Delta H_{j},
\end{aligned}
$$

no qual $\Delta H_{j}=\sum_{k=0}^{\infty}\left[e^{i u k}-1\right] \Delta r(\cdot, k, j)$. Pelo Brémaud (teorema 4 da pg 337) [2], concluímos que

$$
f_{t}=\prod_{j=s+1}^{t}\left(1+\Delta H_{j}\right)
$$

independente de $A$. Portanto

$$
\frac{E\left[\mathbb{1}_{A} Y_{t}\right]}{P(A)}=\frac{E\left[\mathbb{1}_{\Omega} Y_{t}\right]}{P(\Omega)}=E\left[Y_{t}\right]
$$

Assim, concluímos que processo $N$ apresenta incrementos independentes.

Suponha agora que $N$ tenha incrementos independentes, o que implica que $E\left[N\left(\cdot, t_{2}\right)-\right.$ $\left.N\left(\cdot, t_{1}\right) \mid \mathcal{F}_{t_{1}}\right]=E\left[N\left(\cdot, t_{2}\right)-N\left(\cdot, t_{1}\right)\right]$, com $t_{1}<t_{2}$. Logo temos que

$$
\widetilde{N}(\cdot, t)=\sum_{j=1}^{t} E\left[N(\cdot, j)-N(\cdot, j-1) \mid \mathcal{F}_{j-1}\right]=\sum_{j=0}^{t} E[N(\cdot, j)-N(\cdot, j-1)]
$$

o que implica que $\widetilde{N}(\cdot, t)$ é não aleatório.

Lema 2.4.4. Seja $M(s, t)^{u}=\frac{e^{i u(N(\cdot, t)-N(\cdot, s))}}{E\left[e^{i u(N(\cdot, t)-N(\cdot, s))}\right]}, t, s \in \mathbb{N} \operatorname{com} t \geq s$. Então $\left(M(s, t)^{u}\right)_{t \geq s} e ́$ um $(\mathcal{F})_{t \geq s}$-martingale.

Demonstração: Seja $t_{0} \in \mathbb{N}$, tal que $s<t_{0}<t$ então

$$
\begin{aligned}
E\left[M(s, t)^{u} \mid \mathcal{F}_{t_{0}}\right] & =\frac{e^{i u\left(N\left(\cdot, t_{0}\right)-N(\cdot, s)\right)}}{E\left[e^{i u(N(\cdot, t)-N(\cdot, s))}\right]} \frac{E\left[e^{i u\left(N\left(\cdot, t_{0}\right)-N(\cdot, s)\right)}\right]}{E\left[e^{i u(N(\cdot, t)-N(\cdot, s))}\right]} \\
& =\frac{e^{i u\left(N\left(\cdot, t_{0}\right)-N(\cdot, s)\right)}}{E\left[e^{i u\left(N\left(\cdot, t_{0}\right)-N(\cdot, s)\right)}\right]} \\
& =M\left(s, t_{0}\right)^{u}
\end{aligned}
$$


Se além da hipótese de que os incrementos são independentes adicionarmos a hipótese de que são estacionários, o que significa dizer:

Definição 2.4.5. Um processo estocástico $\{N(\cdot, t), t \in \mathbb{N}\}$ discreto é dito ter incrementos estacionários se $P[N(\cdot, t+s)-N(\cdot, t)=k]=P[N(\cdot, s)=k]$, para qualquer $k, t \in \mathbb{N}$.

Teorema 2.4.6. $O$ processo $N$ tem incrementos independentes e estacionários se, e somente se, existe $\lambda \in(0,1)$, tal que $\Delta \widetilde{N}(\cdot, \ell)=\lambda, \forall \ell \in \mathbb{N}$.

Demonstração: Primeiramente mostremos que se $\Delta \widetilde{N}(\cdot, \ell)=\lambda, \lambda \in(0,1)$, então $N$ tem incrementos independentes e estacionários. Mostramos que se $N$ tem incrementos independentes se, e somente, se $\widetilde{N}$ é não aleatório, note que $\Delta \widetilde{N}=\lambda$, isso implica que $\widetilde{N}(\cdot, \ell)=\lambda \ell$, o qual é não aleatório $\operatorname{logo} N$ tem incrementos independentes, assim basta mostramos que $N$ é estacionário

Lembrando que $\mathrm{N}$ não depende da filtragem pelo fato dele ter incrementos independentes.

$$
\begin{aligned}
E\left[N\left(\cdot, t_{1}+1\right)-N\left(\cdot, t_{1}\right)\right] & =\sum_{j=1}^{s} \lambda \\
& =\widetilde{N}(\cdot, s)=E[N(\cdot, 1)], \forall t_{1} \in \mathbb{N} .
\end{aligned}
$$

Assim por indução temos que

$$
E\left[N\left(\cdot, t_{1}+s\right)-N\left(\cdot, t_{1}\right)\right]=E[N(\cdot, s)], \forall t_{1}, s \in \mathbb{N} \text { e } t_{1}<s .
$$

Além disso, definamos $\varphi(t)=E\left[e^{i u(N(\cdot, t))}\right]$ e $\widetilde{M}(t)^{u}=\frac{e^{i u(N(\cdot, t))}}{\varphi(t)}$, lembrando que pelo lema 2.4.4 $\widetilde{M}(t)^{u}$ é um martingale. Queremos mostrar que N é um processo com incrementos estácionarios, todavia devido as propriedades da transformada de fourier temos que é equivalente mostrar que

$$
E\left[e^{i u(N(\cdot, t+s)-N(\cdot, t))}\right]=E\left[e^{i u(N(\cdot, s))}\right], \quad \forall t, s \in \mathbb{N}, \quad u \in \mathbb{R} .
$$

Notemos que 


$$
\begin{aligned}
E\left[e^{i u(N(\cdot, t+s)-N(\cdot, t))}\right] & =E\left[\frac{e^{i u(N(\cdot, t+s))}}{\left.e^{i u(N(\cdot, t))} \frac{\varphi(t+s)}{\varphi(t+s)} \frac{\varphi(t)}{\varphi(t)}\right]}\right. \\
& =E\left[\frac{M_{t+s}^{u}}{M_{t}^{u}} \frac{\varphi(t+s)}{\varphi(t)}\right] \\
& =\frac{\varphi(t+s)}{\varphi(t)} E\left[\frac{M_{t+s}^{u}}{M_{t}^{u}}\right] \\
& =\frac{\varphi(t+s)}{\varphi(t)} \\
& \stackrel{2.21}{=} \varphi(s)
\end{aligned}
$$

Portanto o $\mathrm{N}$ tem incrementos independentes e estácionários.

Mostremos agora que se $\widetilde{N}$ tem incrementos independentes e estacionária então $\Delta \widetilde{N}(\cdot, \ell)=$ $\lambda, \operatorname{com} \lambda \in(0,1)$. Assim basta notarmos

$$
\begin{aligned}
\widetilde{N} & =\sum_{j=1}^{t} E[N(\cdot, j)-N(\cdot, j-1)] \\
& =\sum_{j=1}^{t} E[N(\cdot, 1)] \\
& =\sum_{j=1}^{t} E[N(\cdot, 1)] \\
& =(t) E[N(\cdot, 1)]
\end{aligned}
$$

portanto $\widetilde{N}$ é linear logo o resultado segue, pois basta tomar $\lambda=E[N(\cdot, 1)]$.

Lema 2.4.7. Se $D_{i} \stackrel{i i d}{\sim} G e o(\lambda)$, então $N$ tem incrementos independentes e estácionários.

Demonstração: De fato, como $\Delta \widetilde{N}=\lambda, \lambda \in(0,1)$, pois pela equação 2.14 temos que, se os $D_{i}$ são independentes, então seu compensador é dado por

$$
\widetilde{N}(\cdot, t)=\sum_{\ell=0}^{D_{1}} \frac{P\left(D_{1}=\ell\right)}{P\left(D_{1} \geq \ell\right)}+\cdots+\sum_{\ell=0}^{t-T_{j}} \frac{P\left(D_{1}=\ell\right)}{P\left(D_{1} \geq \ell\right)}
$$

Além disso, temos que

$$
\frac{P\left(D_{1}=\ell\right)}{P\left(D_{1} \geq \ell\right)}=\frac{(1-\lambda)^{k} \lambda}{1-\sum_{l=0}^{k}(1-\lambda)^{l}}=\frac{(1-\lambda)^{k} \lambda}{1-\lambda \frac{(1-\lambda)^{t}-1}{\lambda}}=\lambda
$$

temos, então que: 


$$
\tilde{N}(\cdot, t)=\sum_{\ell=0}^{D_{1}} \lambda+\cdots+\sum_{\ell=0}^{t-T_{j}} \lambda=t \lambda
$$

Teorema 2.4.8. O processo $N$ tem incrementos independentes e estacionários se, e somente se, $D_{i} \sim G e o(\lambda)$.

Demonstração: Pelo lema 2.4.7 temos que se $D_{i} \sim G e o(\lambda)$ então $N$ tem incrementos independentes. Agora suponha que $N$ tem incrementos independentes e estacionário, o que pelo teorema 2.4 .6 basta mostrar que $\Delta \widetilde{N}(\ell)=\lambda$ para algum $\lambda \in(0,1)$ e $\forall \ell \in \mathbb{N}$, então $D_{i} \sim G e o(\lambda)$. De fato,

$$
\begin{aligned}
\Delta \tilde{N}(0) & =\lambda=\frac{P\left(D_{i}=0\right)}{P\left(D_{i} \geq 0\right)}=P\left(D_{i}=0\right) \\
& \Rightarrow P\left(D_{i}=0\right)=\lambda,
\end{aligned}
$$

para $\ell=1$ temos

$$
\begin{aligned}
\Delta \widetilde{N}(1) & =\lambda=\frac{P\left(D_{i}=1\right)}{P\left(D_{i} \geq 1\right)} \\
& \Rightarrow P\left(D_{i}=1\right)=\lambda\left[P\left(D_{i} \geq 1\right)\right]=\lambda\left[1-P\left(D_{i}=0\right)\right]=\lambda[1-\lambda]
\end{aligned}
$$

De forma indutiva temos que

$$
P\left(D_{i}=\ell\right)=\lambda(1-\lambda)^{\ell}, \quad \forall \ell \in \mathbb{N}
$$

Portanto temos que $D_{i} \sim G e o(\lambda)$ e o resultado segue.

Observe que com os resultados demonstrados acima podemos garantir que a única distribuição discreta que satisfaz os incrementos serem independentes e estacionários é a distribuição geométrica, já que dado a função intensidade se define de forma única a distribuição de probabilidades. 


\begin{tabular}{c|c|}
\hline & Capítulo \\
\hline & 3 \\
\hline
\end{tabular}

\section{Distribuição assintótica da estatística de logrank.}

Neste capitulo temos por objetivo de desenvolver uma estratégia para derivarmos o seguinte teste de hipótese.

$$
\left\{\begin{array}{l}
H_{0}: N \text { tem incrementos independentes e estacionários, } \\
H_{1}: N \text { não apresenta incrementos independentes e } \backslash \text { ou estacionários. }
\end{array}\right.
$$

Sobre a hipótese nula, temos que pelos resultados anteriores, que os $D_{i}$ 's são independentes e indenticamente distribuídos, com distribuição comum geométrica.

Desta forma podemos simplificar nosso teste baseado nos resultados apresentados no capítulo anterior, utilizando de forma mais adequada da nossa hipótese nula. Mostramos que quando estamos sobre $H_{0}$ os tempos entre ocorrências de negócios segue uma distribuição geométrica. Assim podemos desenvolver o teste de hipótese baseado apenas nos tempos entre negócios e não mais em todo intervalo, pois o intuíto é verificar se o intervalo entre negócios seguem uma distribuição geométrica de parâmetro $\lambda$. Então como $D_{m} \stackrel{i i d}{\sim} G e o(\lambda)$, podemos fazer uma simplificação no nosso modelo, definindo

$$
F_{m}(i)=\mathbb{1}_{\left\{D_{m} \leq i\right\}}
$$


Notemos que neste caso $F_{m}$ é um submartingale. Portanto podemos encontrar a parte previsível da decomposição de Doob-Meyer utilizando o teorema 2.2.2. Temos que o compensador é dado da seguinte forma

$$
\begin{aligned}
& \widetilde{F}_{m}(i)=\sum_{\ell=0}^{i} \frac{P\left[D_{m}=i\right]}{P\left[D_{m} \geq \ell\right]} \mathbb{1}_{\left\{D_{m} \geq \ell\right\}} . \\
& F(i)=\sum_{m=1}^{n} F_{m}(i)=\sum_{m=1}^{n} \mathbb{1}_{\left\{D_{m} \leq i\right\}}
\end{aligned}
$$

com $n$ sendo o número de instantes em que ocorreram negócios. Como por hipótese $D_{p}$ e $D_{q}$ são independentes para $p \neq q$, então o compensador de $F$ será dado por

$$
\widetilde{F}(i)=\sum_{m=1}^{n} \widetilde{F}_{m}(i)=\sum_{\ell=0}^{i} \sum_{m=1}^{n} \frac{P\left[D_{m}=\ell\right]}{P\left[D_{m} \geq \ell\right]} \mathbb{1}_{\left\{D_{m} \geq \ell\right\}}
$$

como demonstrado no lema 2.4.7. Assim como a função intensidade é definida por

$$
h_{m}(i)=\frac{P\left[D_{m}=i\right]}{P\left[D_{m} \geq i\right]}=\frac{\Delta \tilde{N}_{m}(i)}{V_{m}(i)}
$$

$\operatorname{com} V(i)=\mathbb{1}_{\left\{D_{m} \geq i\right\}}$, então temos que

$$
\widetilde{F}(i)=\sum_{\ell=0}^{i} \sum_{m=1}^{n} \frac{P\left[D_{m}=\ell\right]}{P\left[D_{m} \geq \ell\right]} \mathbb{1}_{\left\{D_{m} \geq i\right\}}=\sum_{\ell=0}^{i} \sum_{m=1}^{n} h(\ell) \mathbb{1}_{\left\{D_{m} \geq \ell\right\}}=\sum_{\ell=0}^{i} h(\ell) V(\ell)
$$

$\operatorname{com} V(i)=\sum_{m=1}^{n} \mathbb{1}_{\left\{D_{m} \geq i\right\}}$. Um estimado empírico para $h$ é dado por

$$
\widehat{h}(i)=\frac{\Delta F(i)}{V(i)} \mathbb{1}_{\{V(i)>0\}}
$$

o qual é conhecido como estimador de kaplan-meier. A estatística de Logrank definida como sendo

$$
\begin{aligned}
L R(i) & =\sum_{d=0}^{i} \frac{\sqrt{n}}{V(d)} \Delta Y(\cdot, d)=\sum_{d=0}^{i} \frac{\sqrt{n}}{V(d)}(\Delta F(\cdot, d)-\Delta \widetilde{F}(\cdot, d)) \\
& =\sum_{d=0}^{i} \sqrt{n}\left(\frac{\Delta F(\cdot, d)}{V(d)}-\frac{h(d) V(d)}{V(d)}\right)=\sum_{d=0}^{i} \sqrt{n}(\widehat{h}(d)-h(d))
\end{aligned}
$$

Portanto sobre $H_{0}$ temos que a estatística de Logrank é dada por 


$$
L R(i)=\sum_{d=0}^{i} \sqrt{n}(\widehat{h}(d)-\lambda)
$$

Teorema 3.0.9. Seja $H_{d}^{n}=\sqrt{n}(\widehat{h}(d)-\lambda)$ então $H_{d}^{n}$ converge em distribuição para $N\left(0, \sigma_{d}^{2}\right)$, para todo $d=0,1, \ldots, n$. Além disso, a estatística de Logrank tem incrementos assintóticamente independentes e portanto converge para uma $N\left(0, \sum_{d=0}^{i} \sigma_{d}^{2}\right)$, com

$$
\sigma_{d}^{2}=\lambda[1-\lambda] \frac{1}{P\left[D_{m} \geq d\right]}=\frac{\lambda[1-\lambda]}{1-P\left[D_{m}<d\right]}=\frac{\lambda}{(1-\lambda)^{d}}
$$

A demonstração do teorema acima foi feita pelo Leão e Ohashi (2011)[13].

O teorema 3.0.9 nos dá a distribuição assintótica da estatística do teste sobre $H_{0}$, para o caso totalmente especificado, que significa dizer que conhecemos o parâmetro da geométrica. Na seção a seguir vamos propor um teste, para o qual não existe a necessidade de conhecermos o parâmetro da distribuição dos tempos entre negócios. Assim se $D_{i}^{\prime} s \stackrel{i i d}{\sim} G e o(\lambda)$ não necesitamos conhecer $\lambda$, utilizando apenas o estimador empírico de kaplan-meier.

\subsection{Hipótese nula não especificada e a conjectura de Simes.}

Na seção anterior apresentamos o caso totalmente especificado. Entretanto em termos práticos este caso raramente ocorre, pois dificilmente teremos a distribuição e seu parâmetro de forma exata. Assim necessitamos de encontrar um método para verificar se a amostra vem de um processo que apresenta incrementos estacionários e independentes, de tal sorte que não precisamos conhecer o parâmetro de forma direta.

Notemos que quando estamos sobre a hipótese nula, temos que $h$ é constante e igual ao parâmetro $\lambda$ da geométrica. Desta forma, baseado nesta informação temos que

$$
\sqrt{n}(\widehat{h}(j)-\widehat{h}(i))
$$

no qual $\widehat{h}$ é definido

$$
\widehat{h}(i)=\frac{\Delta N(i)}{V(i)} \mathbb{1}_{\{V(i)>0\}},
$$

no qual $V(i)$ é definido como

$$
V(i)=\sum_{m=1}^{\infty} \mathbb{1}_{\left\{D_{m} \geq i\right\}} .
$$

Como demonstrado na seção anterior, temos que $\sqrt{n}[\widehat{h}(i)-\lambda]$ converge em distribuição para $N\left(0, \sigma_{i}^{2}\right)$. Assim, pelo fato da estatística de Logrank apresentar incrementos assintóticamente independentes, temos que 


$$
\sqrt{n}[\widehat{h}(i)-\lambda]-\sqrt{n}[\widehat{h}(j)-\lambda]=\sqrt{n}[\widehat{h}(i)-\widehat{h}(j)]
$$

o qual, converge fracamente para uma $N\left(0, \sigma_{i}^{2}+\sigma_{j}^{2}\right)$. Neste caso, não precisamos nos preocupar de forma direta com o valor de $\lambda$, ou mesmo necessitamos estimá-lo.

Assim basta testarmos se

$$
\left\{\begin{array}{c}
H_{0 i}: h(0)=h(i)=\lambda \\
H_{1 i}: h(0) \neq h(i)
\end{array}\right.
$$

para todo $i$, o que implica que

$$
\left\{\begin{array}{c}
H_{0}=\bigcap_{i=1}^{n} H_{0 i}: h(0)=h(1)=\cdots=h(n), \\
H_{1}: h(0) \neq h(i) \text { para algum } i \in\{1,2, \cdots, n\} .
\end{array}\right.
$$

Deste modo se $H_{0}$ não for rejeitada obtemos que $h(i)$ é uma constante para todo $i$. Assim, a amostra tem evidências de vir de um processo com incrementos independentes e estacionários. Entretanto, nessa abordagem precisamos nos atentar a um pequeno infortúnio, o qual é devido ao fato de estarmos fazendo múltiplos testes, e portanto necessitamos de uma estratégia para controlarmos a probabilidade de erro do tipo I. Para contornarmos esse obstáculo usaremos o teste proposto por Hochberg [11] conhecido como familywise error rate (FWER), e também a conjectura de Simes [18]. Um dos métodos de controle da FWER é o a desigualdade de Bonferroni, a qual é definida da seguinte forma. Dado uma coleção de $n$ hipótese nula $H_{0_{1}}, H_{0_{2}}, \ldots, H_{0_{n}}$ com os p-valores correspondentes $P_{1}, P_{2}, \ldots, P_{n}$ e $H_{0}=$ $\cap_{i=1}^{n} H_{0_{i}}$ com nivel de significância $\alpha$

$$
P\left(\cup_{i=1}^{n}\left(P_{i} \leq \alpha / n\right)\right) \leq \alpha
$$

Esse é o teste mais comum, o qual rejeitamos $H_{0}$ se $P_{i} \leq \alpha / n$ para pelo menos um i.

A conjectura de Simes nos concede uma forma de controlarmos a FWER, portanto ela nos concede uma maneira de controlarmos o erro global. A conjectura de Simes é feita da seguinte foma.

1. Ordenamos os p-valores $P_{(1)} \leq \cdots \leq P_{(n)}$

2. Rejeitamos $H_{0}$ se $P_{(i)} \leq i \alpha / n$

Esse método é mais poderoso do que a desigualdade de Bonferroni. Todavia a conjectura de Simes não é válida para qualquer conjunto de estatística do teste, o próprio Simes em sua pesquisa apresentou alguns contra-exemplos. Mas podemos ver no trabalho de Sarkar e Chang [16] que quando o teste apresenta dependência assintóticamente positiva, ou simplesmente dependência postiva, então a conjectura de Simes é válida. 
Assim, mostremos que nossa estatística apresenta dependência assintóticamente positiva, o que implica que a conjectura de Simes é válida.

Teorema 3.1.1. O teste apresenta dependência assintóticamente positiva.

Demonstração: Basta encontarmos a covariância do teste e mostrarmos que ela é positiva. Assim, basta calcularmos $\operatorname{Cov}\left[X_{i}, X_{j}\right], \operatorname{com} X_{i}=\sqrt{n}(\widehat{h}(0)-\widehat{h}(i))$

De fato, temos que $\operatorname{Cov}\left[X_{i}, X_{j}\right]>0$, pois como a covariância é bilinear temos

$$
\begin{aligned}
\operatorname{Cov}\left[X_{i}, X_{j}\right] & =\operatorname{Cov}[\sqrt{n}(\widehat{h}(0)-\widehat{h}(i)), \sqrt{n}(\widehat{h}(0)-\widehat{h}(j))] \\
& =n\{\operatorname{Cov}[\widehat{h}(0), \widehat{h}(0)]-\operatorname{Cov}[\widehat{h}(0), \widehat{h}(j)]-\operatorname{Cov}[\widehat{h}(i), \widehat{h}(0)]+\operatorname{Cov}[\widehat{h}(i), \widehat{h}(j)]\},
\end{aligned}
$$

como $\widehat{h}(i)$ e $\widehat{h}(j)$ são assintóticamente independentes $\forall i, j \in\{0,1,2, \cdots\}$, com $i \neq j$, temos que

$$
\operatorname{Cov}[\widehat{h}(i), \widehat{h}(j)]=\operatorname{Cov}[\widehat{h}(0), \widehat{h}(j)]=\operatorname{Cov}[\widehat{h}(i), \widehat{h}(0)]=0
$$

Portanto, $\operatorname{Cov}\left[X_{i}, X_{j}\right]=\operatorname{Cov}[\widehat{h}(0), \widehat{h}(0)]>0$, desta forma a conjectura de Simes é válida.

Na seção seguinte apresentamos um estudo de simulações.

\subsection{Estudo de Simulação}

Fizemos um estudo de simulação para avaliarmos a estatistica que propomos. Para isso tomamos uma amostra gerada a partir de uma distribuição geométrica de parâmetro $\lambda$, com o intuito de analizarmos a probabilidade do erro do tipo I, desta forma fixamos nossa probabilidade nominal $(\alpha)$ em 0,05 e o tamanho da amostra(TA). Os passos abaixo mostram a arquitetura do algoritmo proposto, o qual foi simulado sobre a hipótese nula.

1. Geramos uma amostra de tamanho TA com distribuição geométrica de parâmetro $\lambda$. Desta forma, estaremos sob a hipótese nula.

2. Realizamos o teste de hipótese com nivel de significância $\alpha$.

3. Verificamos se cometeu o erro do tipo I, isto é, a hipótese nula ser rejeitada dado que ela é verdadeira.

$$
y_{k}=\left\{\begin{array}{c}
1, \text { se } H_{0} \text { for rejeitado ao nivel de significância } \alpha \\
0, \text { caso contrário. }
\end{array}\right.
$$


4. Repetimos os itens anteriores $n=5000$.

5. Assim estimamos a probabilidade de ocorrência do erro do tipo I

$$
\widehat{\alpha}=\frac{1}{n} \sum_{k=1}^{n} y_{k}
$$

\begin{tabular}{cccccccc}
\hline & \multicolumn{7}{c}{$\lambda$} \\
& \multicolumn{7}{c}{$\mathbf{0}$} \\
\cline { 2 - 8 } T.A. & $\mathbf{0 , 2}$ & $\mathbf{0 , 3}$ & $\mathbf{0 , 4}$ & $\mathbf{0 , 5}$ & $\mathbf{0 , 6}$ & $\mathbf{0 , 7}$ & $\mathbf{0 , 8}$ \\
& & & & & & & \\
\hline & & & & & & & \\
100 & 0,054000 & 0,054875 & 0,041375 & 0,055000 & 0,057375 & 0,055750 & 0,044250 \\
200 & 0,053125 & 0,056000 & 0,042250 & 0,057500 & 0,055625 & 0,053500 & 0,047500 \\
300 & 0,051625 & 0,050125 & 0,044625 & 0,048375 & 0,044875 & 0,054250 & 0,047750 \\
400 & 0,050875 & 0,055875 & 0,044375 & 0,054750 & 0,052500 & 0,046500 & 0,052125 \\
500 & 0,050250 & 0,053375 & 0,046500 & 0,054375 & 0,053750 & 0,048750 & 0,051625 \\
600 & 0,058750 & 0,053500 & 0,046750 & 0,052250 & 0,052125 & 0,053750 & 0,047250 \\
700 & 0,052375 & 0,053125 & 0,047375 & 0,048500 & 0,049875 & 0,052375 & 0,048625 \\
800 & 0,049500 & 0,054375 & 0,049250 & 0,051500 & 0,050875 & 0,049875 & 0,051125 \\
1000 & 0,051750 & 0,053125 & 0,049850 & 0,051875 & 0,050250 & 0,050750 & 0,049375 \\
2000 & 0,049125 & 0,050375 & 0,050250 & 0,049500 & 0,050750 & 0,050375 & 0,050475 \\
3000 & 0,050500 & 0,050150 & 0,049950 & 0,050120 & 0,049950 & 0,050275 & 0,050750
\end{tabular}

Tabela 3.1: Tabela de erro do Tipo I, com número de iterações igual a $5 \cdot 10^{3}$.

Observemos que na tabela 3.2 para todos os valores de $\lambda$ a estatística do teste converge para o valor nominal $(\alpha)$, conforme aumentamos o tamanho da amostra.

\subsubsection{Poder do teste}

O poder do teste tem como intenção conhecer o quanto o teste controla o erro do tipo II. Ele é fundamental, pois um teste com alto poder significa alta capacidade de rejeição da nossa hipótese nula dado que ela é falsa. Assim em nosso caso, o teste tem por objetivo detectar se os dados provém de um processo que apresentam incrementos independentes e estacionários. Desta forma, temos que verificar o quanto o teste é poderoso em detectar dados que não vêem de um processo com distribuição entre ocorrências identicamente distribuídos e geométrica. Vamos calcular o poder do teste considerando uma amostra que provém de uma mistura de geométricas. Assim, seja X uma variável aleatória com distribuição Bernoulli de parâmetro $p$, portanto definimos nossa mistura como sendo 


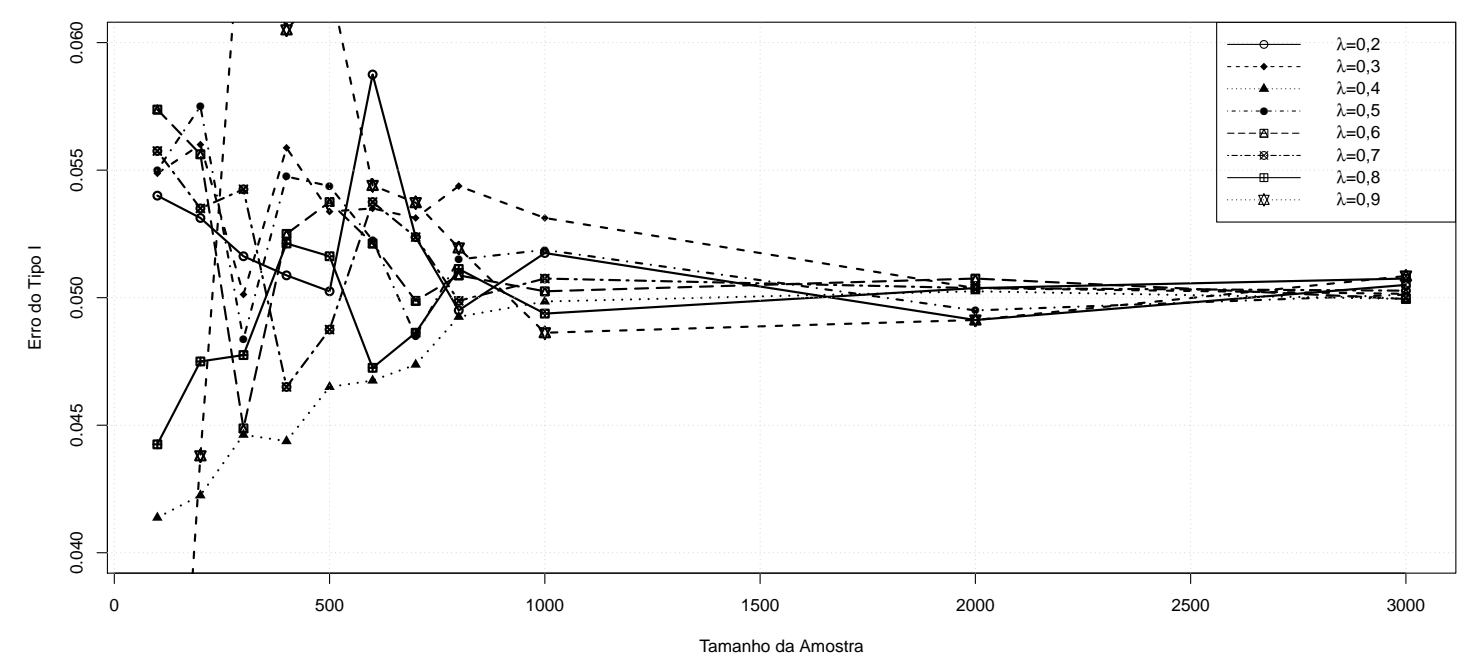

Figura 3.1: A figura mostra a convergência do erro do Tipo I, em relação ao tamanha da amostra. O número de iterações utilizado é igual a $5 \cdot 10^{3}$.

- Se $X=1$, geramos um elemento da amostra a partir de $Y \sim G e o(\lambda)$

- Se $X=0$, geramos um elemento da amostra a partir de $Y \sim G e o(\lambda+\delta)$

Apesar desta mistura de geométrica serem independentes, sua função intensidade não assume valor constante. Portanto pelo teorema 2.4.6 o processo gerado por essa mistura não apresenta incrementos independentes e estacionários.

Parâmetro da Mistura

\begin{tabular}{|c|c|c|c|c|c|c|c|c|c|c|c|c|}
\hline$\delta$ & 0,1 & 0,2 & 0,25 & 0,3 & 0,4 & 0,45 & 0,5 & 0,6 & 0,7 & 0,75 & 0,8 & 0,9 \\
\hline 0,001 & 0,0509 & 0,2136 & 0,3476 & 0,418 & 0,4412 & 0,5098 & 0,5558 & 0,4124 & 0,3028 & 0,2831 & 0,1523 & 0,065 \\
\hline 0,005 & 0,0612 & 0,2467 & 0,4098 & 0,4422 & 0,4891 & 0,5936 & 0,6498 & 0,4834 & 0,3954 & 0,3254 & 0,2343 & 0,1012 \\
\hline 0,01 & 0,1057 & 0,2785 & 0,4149 & 0,4878 & 0,5401 & 0,6322 & 0,7825 & 0,5355 & 0,4136 & 0,3987 & 0,3012 & 0,1512 \\
\hline 0,05 & 0,1863 & 0,3211 & 0,4834 & 0,5143 & 0,5342 & 0,7234 & 0,8354 & 0,5987 & 0,4857 & 0,4521 & 0,3767 & 0,2076 \\
\hline 0,1 & 0,2647 & 0,3887 & 0,5438 & 0,6453 & 0,6071 & 0,8253 & 0,8435 & 0,7082 & 0,5621 & 0,5076 & 0,4333 & 0,3113 \\
\hline 0,15 & 0,3421 & 0,4112 & 0,5641 & 0,6987 & 0,6875 & 0,8765 & 0,9276 & 0,7986 & 0,6349 & 0,5978 & 0,4913 & 0,3987 \\
\hline 0,2 & 0,4623 & 0,4633 & 0,6 & 0,7246 & 0,7182 & 0,9102 & 0,9996 & 0,8142 & 0,6998 & 0,6591 & 0,5543 & 0,4316 \\
\hline 0,25 & 0,521 & 0,5016 & 0,6244 & 0,809 & 0,7724 & 0,9834 & 1 & 0,8899 & 0,7432 & 0,7383 & 0,6123 & 0,5161 \\
\hline 0,3 & 0,5613 & 0,5987 & 0,687 & 0,8989 & 0,8243 & 1 & 1 & 0,9567 & 0,8768 & 0,8763 & 0,6957 & 0,6098 \\
\hline 0,35 & 0,6036 & 0,6231 & 0,7587 & 0,9387 & 0,8991 & 1 & 1 & 1 & 0,9209 & 0,9089 & 0,7812 & 0,6978 \\
\hline 0,4 & 0,6938 & 0,7765 & 0,8324 & 0,9908 & 0,9456 & 1 & 1 & 1 & 0,9135 & 0,9678 & 0,8749 & 0,7691 \\
\hline 0,45 & 0,7722 & 0,8402 & 0,9132 & 1 & 0,9745 & 1 & 1 & 1 & 1 & 1 & 0,9412 & 0,8135 \\
\hline 0,5 & 0,8431 & 0,8856 & 0,9654 & 1 & 1 & 1 & 1 & 1 & 1 & 1 & 0,9912 & 0,8913 \\
\hline 0,55 & 0,9041 & 0,978 & 1 & 1 & 1 & 1 & 1 & 1 & 1 & 1 & 1 & 0,9153 \\
\hline 0,6 & 0,9445 & 0,9984 & 1 & 1 & 1 & 1 & 1 & 1 & 1 & 1 & 1 & 0,9816 \\
\hline 0,65 & 1 & 1 & 1 & 1 & 1 & 1 & 1 & 1 & 1 & 1 & 1 & 1 \\
\hline
\end{tabular}

Tabela 3.2: Tabela do poder do teste 

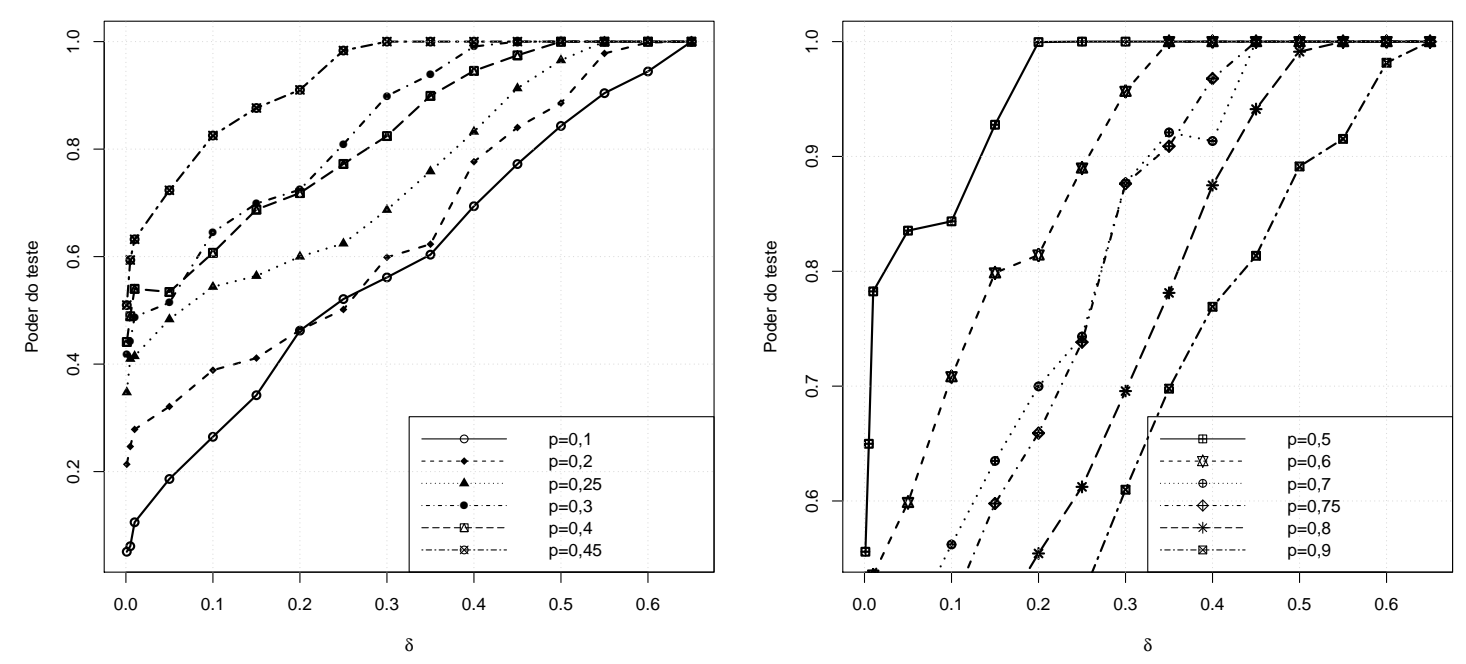

Figura 3.2: A figura ilustra o poder do teste em relação a $\delta$ e o parâmetro da bernoulli $(p)$.

Na tabela 3.2 podemos ver que quando o parâmetro da bernoulli se aproxima de 0 ou se aproxima de 1, a mistura entre as geométricas é menor, desta forma torna-se mais difícil indentifica-la. Todavia quando o parâmetro está próximo de 0,5 a mistura é maior e portanto o poder do teste se torna maior. No estudo de simulação utilizamos $\lambda=0,3$, portanto $\delta$ tem que ser menor que 0,7 . Pois caso contrário teriamos que teriamos que $\lambda+\delta \geq 1$, o que não pode, pois esse é o parâmetro da geométrica. Utilizamos o tamanho da amostra igual a 4000. 


\begin{tabular}{l|l|}
\hline & Capítulo \\
\cline { 2 - 4 } & \\
\hline
\end{tabular}

Neste capítulo encontramos a distribuição exata do teste. Claro que a distribuição exata nos fornece uma informação mais precisa do que a assintótica. Entretanto devido a dificuldades que se deve ao tempo computacional, ela se mostra viável apenas para amostra pequenas. Na qual o poder do teste para distribuição assintótica é muito pequeno, como podemos ver na tabela 4.1 .

Na tabela buscamos fixar todos os parâmetros, variando apenas o tamanho da amostra. Fixamos $\delta=0,45$, o parâmetro da Bernoulli $p=0,5$ e número de iterações igual a 5 mil. Desta forma, encontramos em qual momento o uso da distribuição assintótica se torna inviável. Note que para amostras menores que 60 o poder do teste se torna muito pequeno, neste caso não se torna factível o uso da distribuição assintótica.

Na seção a seguir vamos buscar encontrar a distribuição exata do teste. 
Tamanho da Amostra Poder do teste

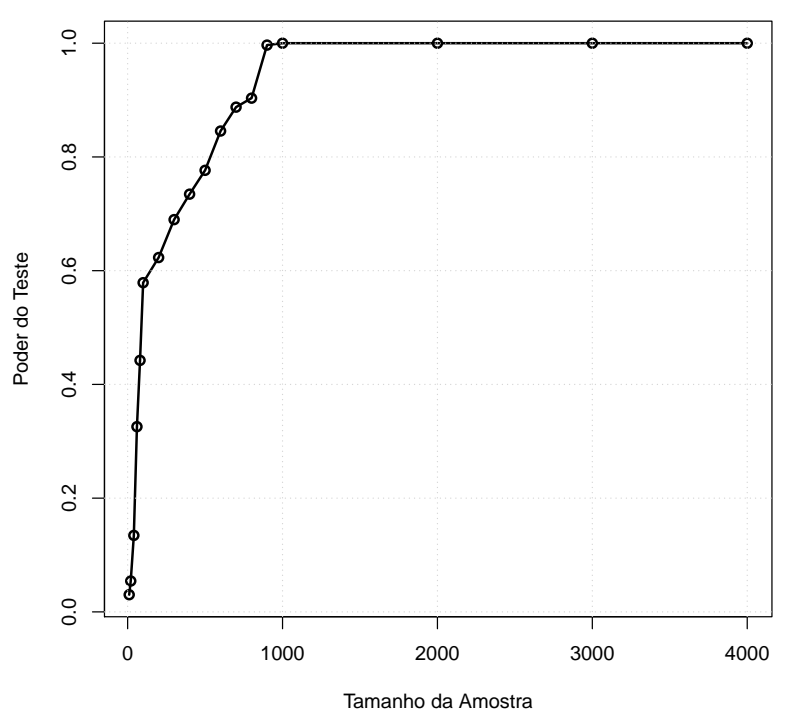

$\begin{array}{cc}10 & 0,03023 \\ 20 & 0,0543 \\ 40 & 0,1345 \\ 60 & 0,3257 \\ 80 & 0,4423 \\ 100 & 0,5789 \\ 200 & 0,6231 \\ 300 & 0,6898 \\ 400 & 0,7346 \\ 500 & 0,7765 \\ 600 & 0,8456 \\ 700 & 0,8876 \\ 800 & 0,9034 \\ 900 & 0,9967 \\ 1000 & 1 \\ 2000 & 1 \\ 3000 & 1 \\ 4000 & 1\end{array}$

Figura 4.1: Fixamos todos os parâmetros, variando apenas o tamanho da amostra, fixamos $\delta=0,45$, o parâmetro da bernoulli $p=0,5$ e número de iterações igual a $5 \cdot 10^{3}$. 


\subsection{Distribuição exata do teste.}

Seja $D=\left\{D_{1}, D_{2}, \cdots, D_{n}\right\}$; onde $D_{i} \stackrel{i i d}{\sim} G e o(p)$. Assim seja

$$
\widehat{h}(\ell)=\frac{\Delta N(\ell)}{V(\ell)}
$$

no qual,

$$
\begin{gathered}
\Delta N(\ell)=\sum_{m=1}^{n} \mathbb{1}_{\left\{D_{m}=\ell\right\}} \\
V(\ell)=\sum_{m=1}^{n} \mathbb{1}_{\left\{D_{m} \geq \ell\right\}} .
\end{gathered}
$$

Observe que $\Delta N(\ell) \sim B\left(n, P\left[D_{m}=\ell\right]\right)$ e $V(\ell) \sim B\left(n, P\left[D_{m} \geq \ell\right]\right)$, além disso temos que $\Delta N(i) \mid V(i) \sim B(V(i), h(i)), \mathrm{com}$

$$
h(i)=\frac{P\left[D_{m}=i\right]}{P\left[D_{m} \geq i\right]}
$$

Assim temos que:

$$
\begin{aligned}
& P\left[\frac{\Delta N(\ell)}{V(\ell)}=\frac{a}{b}\right]=P[\Delta N(\ell)=a ; V(\ell)=b]= \\
& \left(\begin{array}{c}
b \\
a
\end{array}\right) h^{a}(\ell)[1-h(\ell)]^{(b-a)}\left(\begin{array}{c}
n \\
b
\end{array}\right)\left(P\left[D_{m} \geq \ell\right]\right)^{b}\left(P\left[D_{m} \geq \ell\right]\right)^{n-b}
\end{aligned}
$$

Por outro lado temos que:

$$
\widetilde{h}=\frac{n}{n+\sum_{m=1}^{n} D_{m}}
$$

no qual, $\widetilde{h}$ é o estimador de máxima verossimilhança (EMV) do parâmetro $\mathrm{p}$, como $D_{m} \stackrel{i i d}{\sim}$ $G e o(p)$ então $D^{n}=\sum_{m=1}^{n} D_{m} \sim B N(n, 1-p)$.

Desta forma,

$$
P\left[\widetilde{h}=\frac{n}{n+c}\right]=P\left[\frac{n}{n+D^{n}}=\frac{n}{n+c}\right]=P\left[D^{n}=c\right] .
$$

Queremos encontrar a distribuição exata do teste. O nosso teste é dado da seguinte forma: 


$$
\begin{aligned}
& P\left[\frac{\Delta N(i)}{V(i)}-\widetilde{h}=\frac{a}{b}-\frac{n}{n+c}\right]=P\left[\frac{\Delta N(i)}{V(i)}=\frac{a}{b} ; \widetilde{h}=\frac{n}{n+c}\right]= \\
& P\left[\frac{\Delta N(i)}{V(i)}=\frac{a}{b} ; D^{n}=c\right]=P\left[\Delta N(i)=a ; V(i)=b ; D^{n}=c\right]= \\
& P[V(i)=b] P[\Delta N(i)=a \mid V(i)=b] P\left[D^{n}=c \mid \Delta N(i)=a ; V(i)=b\right],
\end{aligned}
$$

$\operatorname{com} a, b, c \in \mathbb{N}, \quad 0 \leq a \leq b \leq n$. Assim se $d \in \operatorname{Im}(\widehat{h}(i)-\widetilde{h})$ então $d=\frac{a}{b}-\frac{n}{n+c}$, para algum $a, b, c \in \mathbb{N}, \quad 0 \leq a \leq b \leq n$.

Lema 4.1.1. Dado $d \in \operatorname{Im}(\widehat{h}(i)-\widetilde{h})$ e n então existem únicos $a, b, c \in \mathbb{N} ;$ tal que a/b é uma fração irredutível e $\frac{a}{b}-\frac{n}{n+c}=d$, ou seja, a função $f_{n}: \mathbb{N} \times \mathbb{N} \rightarrow \mathbb{N}, \operatorname{com} f_{n}(k, c)=k-\frac{n}{n+c}$ é injetora, com $k=a / b$.

Demonstração: Suponha que exista $\left(k_{1}, c_{1}\right)$ e $\left(k_{2}, c_{2}\right)$ tal que $k_{1} \neq k_{2}$ e $c_{1} \neq c_{2}$ e ainda assumimos sem perda de generalidade que $k_{1}<k_{2}$; com $k_{1}=a_{1} / b_{1}$ e $k_{2}=a_{2} / b_{2}$ frações irredutíveis. Como $k_{1}<k_{2} \Rightarrow c_{2}<c_{1}$, pois $d=k_{1}-\frac{n}{n+c_{1}}=k_{2}-\frac{n}{n+c_{2}}$

$$
\begin{aligned}
k_{1}-\frac{n}{n+c_{1}} & =k_{2}-\frac{n}{n+c_{2}} \\
& \Rightarrow k_{1}-k_{2}=\frac{n\left(n+c_{2}\right)-n\left(n+c_{1}\right)}{\left(n+c_{1}\right)\left(n+c_{2}\right)} \\
& \Rightarrow\left(k_{1}-k_{2}\right)\left(n+c_{1}\right)\left(n+c_{2}\right)=n\left(c_{2}-c_{1}\right) .
\end{aligned}
$$

Notemos agora que

$$
n=\frac{k_{1}-k_{2}}{c_{2}-c_{1}}\left(n+c_{1}\right)\left(n+c_{2}\right)
$$

é claro que $\left(n+c_{1}\right)\left(n+c_{2}\right)>n$ o que implica que:

$$
\frac{k_{1}-k_{2}}{c_{2}-c_{1}}<1 \Rightarrow\left(k_{1}-k_{2}\right)<\left(c_{2}-c_{1}\right)
$$

Além disso

$$
\begin{aligned}
\left(k_{1}-k_{2}\right)\left(n+c_{1}\right)\left(n-c_{2}\right)+n\left(c_{1}-c_{2}\right) & =\left(k_{1}-k_{2}\right)\left[n^{2}+n c_{2}+n c_{1}+c_{1} c_{2}\right]+n\left(c_{1}-c_{2}\right) \\
& =n^{2}\left(k_{1}-k_{2}\right)+n\left[\left(c_{1}+c_{2}\right)\left(k_{1}-k_{2}\right)\right. \\
& \left.+\left(c_{1}-c_{2}\right)\right]+\left(c_{1} c_{2}\right)\left(k_{1}-k_{2}\right) \\
& =0 .
\end{aligned}
$$

Observe que 


$$
\begin{gathered}
n^{2}\left(k_{1}-k_{2}\right)<0 \\
c_{1} c_{2}\left(k_{1}-k_{2}\right)<0 .
\end{gathered}
$$

Se mostrarmos que $n\left[\left(c_{1}+c_{2}\right)\left(k_{1}-k_{2}\right)+\left(c_{1}-c_{2}\right)\right]<0$, então teremos um absurdo. Assim como $k_{1}-k_{2}<c_{2}-c_{1}$ temos que

$$
\begin{aligned}
n\left[\left(c_{1}+c_{2}\right)\left(k_{1}-k_{2}\right)+\left(c_{1}-c_{2}\right)\right] & <n\left[\left(c_{1}+c_{2}\right)\left(c_{2}-c_{1}\right)+\left(c_{1}-c_{2}\right)\right] \\
& <n\left[\left(c_{1}^{2}-c_{1}^{2}\right)+\left(c_{1}-c_{2}\right)\right] \\
& <0
\end{aligned}
$$

pois

$$
\begin{aligned}
c_{2}^{2}-c_{1}^{2}+c_{1}-c_{2} & =c_{2}\left(c_{2}-1\right)+c_{1}\left(1-c_{1}\right) \\
& <c_{1}\left(c_{2}-1\right)+c_{1}-c_{1}^{2} \\
& =c_{1} c_{2}-c_{1}+c_{1}-c_{1}^{2} \\
& =c_{1} c_{2}-c_{1}^{2} \\
& <0 .
\end{aligned}
$$

O que é um absurdo assim temos que $k_{1}=k_{2}$ e $c_{1}=c_{2}$. Lembremo-nos apenas que se $k_{1}=k_{2}$ implica necessariamente $c_{1}=c_{2}$.

Tendo mostrado que $d$ é único para $a$ e $b$ irredutíveis, basta apenas calcularmos $P\left[D^{n}=\right.$ $c \mid \Delta N(i)=a ; V(i)=b]$. Entretanto para facilitarmos nossos cálculos vamos mensurar as seguintes probabilidades

Lema 4.1.2. A distribuição de

$$
\begin{gathered}
P\left[D^{n}=c \mid \Delta N(i)=a\right]=\left(\begin{array}{c}
n \\
a
\end{array}\right) P\left[D^{n-a}=c-a i \mid D_{k} \neq i \forall k \in\{1, \cdots, n-a\}\right] \\
P\left[D^{n}=c \mid V(i)=b\right]=\left(\begin{array}{c}
n \\
b
\end{array}\right)\left(\begin{array}{c}
n-a \\
b-a
\end{array}\right) \sum_{j_{1}=0}^{i-1} \cdots \sum_{j_{n-b}=0}^{i-1} \sum_{p_{1}=i}^{c} \cdots \sum_{p_{b-1}=i}^{c} P(A) \\
P(A)=\left(P\left[D_{1}=c-a i-\sum_{k=1}^{n-b} j_{k}-\sum_{t=1}^{b} p_{t}\right] \prod_{k=1}^{n-b} P\left[D_{1}=j_{k}\right] \prod_{t=1}^{b} P\left[D_{1}=p_{t}\right]\right)
\end{gathered}
$$

Demonstração: Primeiramente calcularmos 


$$
\begin{aligned}
P\left[D^{n}\right. & =c \mid \Delta N(i)=a]=\left(\begin{array}{c}
n \\
a
\end{array}\right) P\left[D^{n-a}=c-a i \mid \Delta N(i)=0\right] \\
& =\left(\begin{array}{c}
n \\
a
\end{array}\right) P\left[D^{n-a}=c-a i \mid D_{k} \neq i \forall k \in\{1, \cdots, n-a\}\right],
\end{aligned}
$$

no qual

$$
\begin{aligned}
& P\left[D^{n-a}=c-a i \mid D_{k} \neq i \forall k \in\{1, \cdots, n-a\}\right]= \\
& \sum_{j=0}^{n-(a+1)}\left(\begin{array}{c}
n-a \\
j
\end{array}\right)(-1)^{j} P\left[D^{n-(a+j)}=c-(a+j) i\right] P\left[D_{1}=i\right]^{j}
\end{aligned}
$$

Agora calculemos

$$
P\left[D^{n}=c \mid V(i)=b\right]
$$

Se $c \geq b i$ então temos que:

$$
\begin{aligned}
P\left[D^{n}\right. & =c \mid V(i)=b]=P\left[D^{n}=c \mid E\right] \\
& =\left(\begin{array}{c}
n \\
b
\end{array}\right)\left(\begin{array}{c}
n-a \\
b-a
\end{array}\right) \sum_{j_{1}=0}^{i-1} \cdots \sum_{j_{n-b}=0}^{i-1} \sum_{p_{1}=i}^{c} \cdots \sum_{p_{b-1}=i}^{c} P(A)
\end{aligned}
$$

no qual,

$$
\begin{aligned}
& E=\left\{D^{n} \geq b i ; D_{k} \geq i \forall k \in\{1, \cdots, b\} ; D_{j}<i \forall j \in\{1, \cdots, n-b\}\right\} \\
& P(A)=\left(P\left[D_{1}=c-a i-\sum_{k=1}^{n-b} j_{k}-\sum_{t=1}^{b} p_{t}\right] \prod_{k=1}^{n-b} P\left[D_{1}=j_{k}\right] \prod_{t=1}^{b} P\left[D_{1}=p_{t}\right]\right)
\end{aligned}
$$

$\operatorname{com} c-\left(\sum_{k=1}^{n-b} j_{i}+\sum_{t=1}^{b} p_{t}+a i\right) \geq i$, se $c-\left(\sum_{k=1}^{n-b} j_{k}+\sum_{t=1}^{b} p_{t}+a i\right)<i$ então $P\left[D^{n}=\right.$ $c \mid V(i)=b]:=0$. Além disso se $c<b i$, então também é $P\left[D^{n}=c \mid V(i)=b\right]:=0$.

Lema 4.1.3. A distribuição de probabilidade de $P\left[D^{n}=c \mid V(i)=b ; \Delta N(i)=a\right]$ é igual a 


$$
\begin{gathered}
\left(\begin{array}{l}
n \\
a
\end{array}\right)\left(P\left(D_{1}=i\right)\right)^{a}\left(\sum_{j_{1}=1}^{i-1} \ldots \sum_{j_{n-b}=0}^{i-1} \sum_{p_{1}=i+1}^{c} \ldots \sum_{j_{b-a-1}=i+1}^{c} P(B)\right) \\
P(B)=P\left[D_{1}=c-\sum_{k=1}^{n-b} j_{k}-\sum_{t=1}^{b-a-1} p_{t}\right] \prod_{k=1}^{n-b} P\left[D_{1}=j_{k}\right] \prod_{t=1}^{b-a-1} P\left[D_{1}=p_{t}\right]
\end{gathered}
$$

Demonstração: Com o lema 4.1.2 em mãos estamos aptos a encontrar a probabilidade $P\left[D^{n}=c \mid V(i)=b ; \Delta N(i)=a\right]$. Desta forma temos que

$$
\begin{aligned}
P\left[D^{n}\right. & =c \mid V(i)=b ; \Delta N(i)=a]=P\left[D^{n}=c \mid D\right] \\
& =\left(\begin{array}{c}
n \\
a
\end{array}\right)\left(P\left(D_{1}=i\right)\right)^{a}\left(\sum_{j_{1}=1}^{i-1} \cdots \sum_{j_{n-b}=0}^{i-1} \sum_{p_{1}=i+1}^{c} \ldots \sum_{j_{b-a-1}=i+1}^{c} P(B)\right)
\end{aligned}
$$

no qual

$$
\begin{gathered}
P(B)=P\left[D_{1}=c-\sum_{k=1}^{n-b} j_{k}-\sum_{t=1}^{b-a-1} p_{t}\right] \prod_{k=1}^{n-b} P\left[D_{1}=j_{k}\right] \prod_{t=1}^{b-a-1} P\left[D_{1}=p_{t}\right] \\
D=\left\{D^{n} \geq b i ; D_{k}>i \forall k \in\{1, \cdots, b-a\} ;\right. \\
\left.D_{j}<i \forall j \in\{1, \cdots, n-b-a\} ; D_{p}=i \forall p \in\{1, \cdots, a\}\right\}
\end{gathered}
$$

se $c \geq b i$ e $c-\left(\sum_{k=1}^{n-b} j_{k}+\sum_{t=1}^{b-a-1} p_{t}\right)>i$. Caso contrário $P\left[D^{n}=c \mid V(i)=b ; \Delta N(i)=a\right]:=0$.

Teorema 4.1.4. A distribuição de probabilidade de

$$
P\left[\frac{\Delta N(i)}{V(i)}-\widetilde{h}\right]=P[V(i)=b] P[\Delta N(i)=a \mid V(i)=b] P\left[D^{n}=c \mid \Delta N(i)=a ; V(i)=b\right],
$$

é definida $V(\ell) \sim B\left(n, P\left[D_{m} \geq \ell\right]\right)$ e $\Delta N(\ell) \sim B\left(n, P\left[D_{m}=\ell\right]\right) e$

$$
P\left[D^{n}=c \mid \Delta N(i)=a ; V(i)=b\right]
$$

é denotada pelo lema 4.1 .3

Demonstração: Basta usar os lemas anteriores e o resultado segue.

Para analisar a distribuição exata do teste encontrada, fizemos um teste empírico, no qual tomamos uma amostramos de tamanho $n=18$ e calcumaos de maneira empírica a probabilidade desta acontecer. 
Com esse resultado empírico em mãos calculamos a diferença entre ele e a distribuição proposta. Essa diferença foi da ordem de $10^{-6}$, para todas as amostras testadas.

Note que para realizarmos o teste de hipótese, basta tomarmos nossa amostra e verificarmos qual a probabilidade dela acontecer, utilizando da distribuição do teorema 4.1.4. Com essa probabilidade em mãos, verificamos se ela está acima de um valor aceitável estipulado. Se isso acontecer então não rejeitamos nossa hipótese nula, caso contrário a rejeitamos. 


\begin{tabular}{|l|l|}
\hline & Capítulo \\
\cline { 2 - 2 } & 5 \\
\hline
\end{tabular}

Teste para a PETR4

Na seção 1.1, apresentamos uma breve descrição dos dados a serem utilizados. Nesta seção vamos aplicar os testes de hipóteses aos dados referentes ao tempo entre ocorrência de negócios para as ações da Petrobrás. Com nossos dados sendo do dia 14 de janeiro de 2013 ao dia 18 de janeiro de 2013.

A figura 5.1 exibe o gráfico da distribuição de frequência do tempo entre ocorrência de negócios com sua respectiva tabela. A frequência relativa de cada um dos dias do período estudado, se encontram na figura 5.1

As tabelas 5.25 .3 5.1 mostram as distribuições de frequências do tempo entre ocorrência de negócios. A primeira coluna de cada dia representa o intervalo de tempo apresentado e a segunda representa a frequência de negócios ocorridos. Alguns dados não aparecem nas tabelas, pois foram condensados em uma única linha. As células que apresentam um sinal de + que representa os tempos entre negócios iguais ou superiores ao descrito nas respectivas células. 
$14 / 01 / 2013$

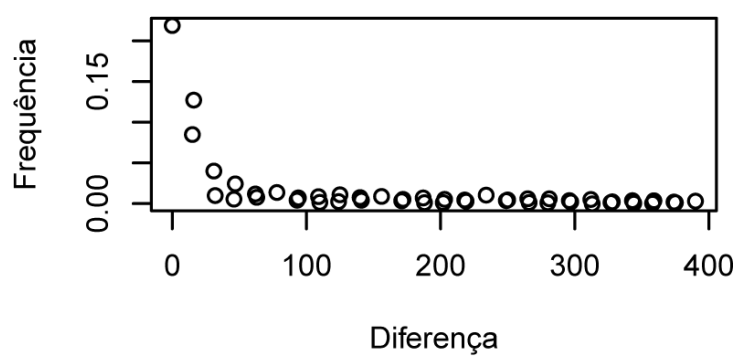

$16 / 01 / 2013$

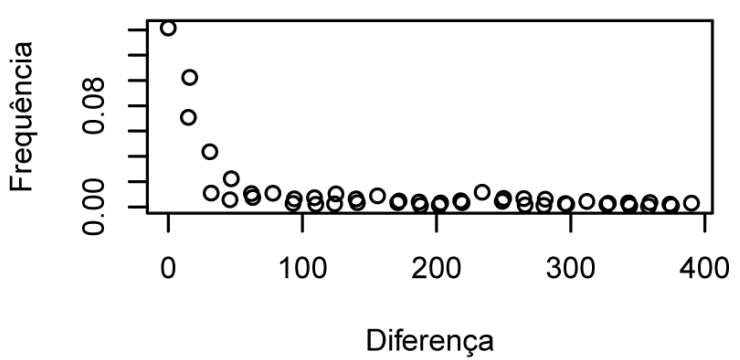

$15 / 01 / 2013$

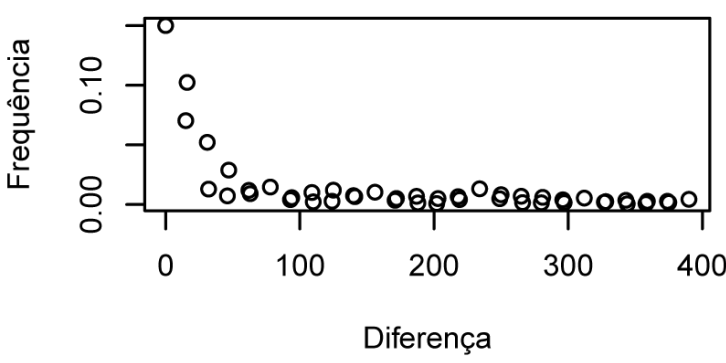

$17 / 01 / 2013$

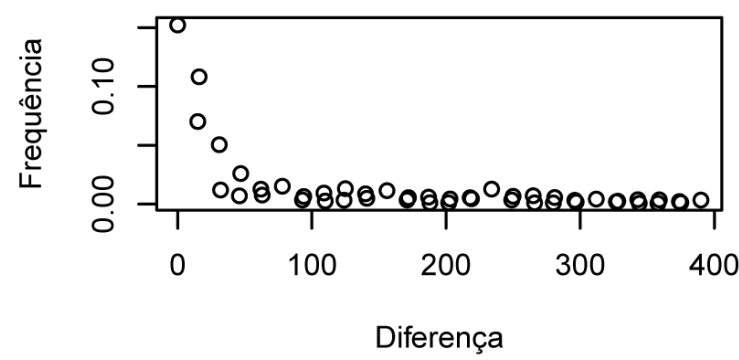

$18 / 01 / 2013$

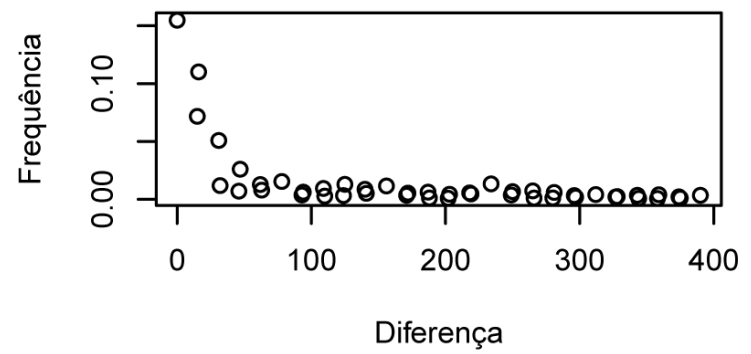

Figura 5.1: Distribuição de frequência do tempo de ocorrência de negócio da PETR4 do dia 14/02/2013 ao dia 18/02/2013, na tabela a palavra Diferença refere-se ao tempo entre ocorrências de negócio. 


\begin{tabular}{ccc}
\hline & & \\
Diferença & Frequência & Acumulada \\
\hline 0 & 0,219116456 & 0,219116456 \\
10 & 0,304291755 & 0,523408212 \\
13 & 0,431784782 & 0,955192994 \\
18 & 0,472093093 & 1,427286087 \\
22 & 0,482290136 & 1,909576223 \\
26 & 0,488078457 & 2,39765468 \\
37 & 0,512671325 & 2,910326005 \\
42 & 0,524817803 & 3,435143808 \\
43 & 0,532795489 & 3,967939298 \\
78 & 0,546771436 & 4,514710734 \\
93 & 0,551270132 & 5,065980866 \\
94 & 0,558618001 & 5,624598866 \\
109 & 0,567495426 & 6,192094293 \\
110 & 0,56941487 & 6,761509162 \\
124 & 0,572264043 & 7,333773206 \\
125 & 0,583420808 & 7,917194014 \\
140 & 0,591158564 & 8,508352578 \\
141 & 0,595627268 & 9,103979846 \\
$142+$ & 1 & 10,10397985 \\
& & \\
\hline
\end{tabular}

\begin{tabular}{ccc}
\hline & & \\
Diferença & Frequência & Acumulada \\
\hline 0 & 0,15025564 & 0,150255637 \\
15 & 0,07054842 & 0,220804061 \\
16 & 0,10258579 & 0,323389855 \\
31 & 0,05234119 & 0,375731048 \\
32 & 0,01313128 & 0,388862324 \\
46 & 0,00743002 & 0,396292346 \\
47 & 0,02905801 & 0,425350351 \\
62 & 0,01206459 & 0,437414941 \\
63 & 0,00930592 & 0,446720859 \\
70 & 0,01486004 & 0,461580903 \\
83 & 0,00430353 & 0,46588443 \\
104 & 0,00595873 & 0,47184316 \\
109 & 0,01029904 & 0,4821422 \\
110 & 0,00246441 & 0,484606613 \\
124 & 0,00294258 & 0,487549196 \\
125 & 0,0122485 & 0,499797697 \\
130 & 0,00765072 & 0,507448413 \\
145 & 0,00676794 & 0,514216353 \\
$150+$ & 0,48578365 & 1 \\
& & \\
\hline
\end{tabular}

Figura 5.2: Tabela da frequência relativa dos dias 14 e 15 de fevereiro de 2013.

\begin{tabular}{ccc}
\hline & & \\
Diferença & Frequência & Acumulada \\
\hline 0 & 0,1417004 & 0,141700405 \\
13 & 0,0710161 & 0,212716533 \\
16 & 0,1026083 & 0,315324882 \\
18 & 0,0438043 & 0,359129223 \\
23 & 0,0111502 & 0,370279419 \\
26 & 0,0058406 & 0,376119997 \\
27 & 0,0224331 & 0,398553129 \\
62 & 0,010752 & 0,409305104 \\
63 & 0,0076989 & 0,417004049 \\
78 & 0,0110175 & 0,428021504 \\
103 & 0,0029867 & 0,431008164 \\
108 & 0,0065707 & 0,437578815 \\
109 & 0,0074335 & 0,445012278 \\
118 & 0,0022566 & 0,447268866 \\
124 & 0,0025884 & 0,449857304 \\
129 & 0,0104865 & 0,460343798 \\
140 & 0,0065043 & 0,466848079 \\
153 & 0,0035176 & 0,4703657 \\
$155+$ & 0,5296343 & 1 \\
& & \\
\hline
\end{tabular}

\begin{tabular}{ccc}
\hline & & \\
Diferença & Frequência & Acumulada \\
\hline 0 & 0,1523999 & 0,152399901 \\
9 & 0,0703805 & 0,222780403 \\
16 & 0,108381 & 0,331161403 \\
21 & 0,0506839 & 0,381845312 \\
22 & 0,012186 & 0,394031335 \\
31 & 0,0072121 & 0,401243472 \\
41 & 0,0261626 & 0,427406118 \\
47 & 0,0128824 & 0,440288485 \\
56 & 0,0079085 & 0,448196966 \\
78 & 0,0153693 & 0,463566277 \\
93 & 0,0036807 & 0,467246953 \\
114 & 0,006665 & 0,473911962 \\
119 & 0,0095996 & 0,483511564 \\
126 & 0,0028351 & 0,48634668 \\
149 & 0,0035315 & 0,48987814 \\
154 & 0,01333 & 0,503208157 \\
165 & 0,008953 & 0,512161154 \\
173 & 0,0051231 & 0,517284258 \\
$178+$ & 0,4827157 & 1 \\
& & \\
\hline
\end{tabular}

Figura 5.3: Tabela da frequência relativa dos dias 16 e 17 de fevereiro de 2013. 


\begin{tabular}{ccc}
\hline & & \\
Diferença & Frequência & Acumulada \\
\hline 0 & 0,1549857 & 0,154985691 \\
8 & 0,0719376 & 0,226923293 \\
12 & 0,1103193 & 0,337242579 \\
18 & 0,0510634 & 0,388305931 \\
44 & 0,0120644 & 0,40037035 \\
46 & 0,0072387 & 0,407609001 \\
67 & 0,026205 & 0,43381404 \\
72 & 0,0129622 & 0,446776275 \\
79 & 0,0081365 & 0,454912743 \\
86 & 0,0155996 & 0,470512317 \\
96 & 0,003928 & 0,474440267 \\
99 & 0,0063969 & 0,480837215 \\
107 & 0,0095954 & 0,490432636 \\
110 & 0,002974 & 0,493406655 \\
128 & 0,0034229 & 0,496829583 \\
134 & 0,0131306 & 0,509960159 \\
140 & 0,0088659 & 0,518826104 \\
146 & 0,0053308 & 0,524156894 \\
$162+$ & 0,4758431 & 1 \\
& & \\
\hline
\end{tabular}

Tabela 5.1: Tabela da frequência relativa do dia 18 de fevereiro de 2013. 


\begin{tabular}{cc}
\hline & \\
Dias & p-valores \\
\hline $14 / 01 / 2013$ & $4,065077 \cdot 10^{-10}$ \\
$15 / 01 / 2013$ & $3,785256 \cdot 10^{-14}$ \\
$16 / 01 / 2013$ & $1,483388 \cdot 10^{-19}$ \\
$17 / 01 / 2013$ & $5,510359 \cdot 10^{-22}$ \\
$18 / 01 / 2013$ & $2,106906 \cdot 10^{-23}$ \\
& \\
\hline
\end{tabular}

Tabela 5.2: Tabela dos p-valores de cada um dos dias do período estudado.

Após termos apresentado os dados, aplicamos o teste de hipótese. Testamos cada um dos dias apresentado na tabela 5.2 . Os p-valores associados são pequenos e portanto existem fortes indícios de que os dados não apresentem incrementos independentes e estacionário. 



\section{Conclusão}

Modelamos o processo pontual do tempo entre negócios em um cenário de análise de ultra-frequência. Além disso encontramos a distribuição do teste para estatistica de logrank toltamente especificada e também desenvolvemos um teste para driblarmos a necessidade de conhecer o parâmetro. O teste de hipótese apresentado é um teste de good-of-fit, encontramos a distribuição exata do teste para a estatística de logrank com o uso do estimador de máxima verossimilhança. Buscamos encontrar a distribuição da função intensidade, que caracteriza o tempo entre negócios dos nossos dados definindo sua intensidade. O tempo entre transações é importante para prever liquidez do ativo, bem como custo do papel. 



\section{Referências Bibliográficas}

[1] L. Bauwens and P. Giot. Econometric Modelling of Stock Market Intraday Activity. Kluwer Academic Publishers, Boston, Dordrecht and London., 2001.

[2] Pierre Brémaud. Point Processes and Queues, Martingale Dynamics. Springer-Verlag, 1th edition, 1981.

[3] C Dellacherie and PA Meyer. Probabilities and potential a, chapter i to iv, 1975.

[4] R. F. Engle. New fronteirs for arch models. Journal of Applied Econometrics, 17:425-446, 2002.

[5] Robert F. Engle and Jeffrey R. Russel. Autoregressive conditional duration: a new model for irregularly spaced transaction data. Econometrica, 66:11271162, 1998.

[6] Scott D. Grimshaw, James McDonald, Grant R. McQueen, and Steven Thorley. Estimating hazard functions for discrete lifetimes. Communications in Statistics - Simulation and Computation, 34:451-463, 2005.

[7] J. Hasbrouck. Measuring the information content of stock trades. Journal of Finance, 46:179-207, 1991.

[8] N. Hautsch. Modelling Irregularly Spaced Financial Data. Springer, Berlin., 2004 .

[9] A. G. Hawkes. Spectra of some self-exciting and mutually exciting point processes. Biometrika, 58:83-90, 1971.

[10] A. Heinen and E. Rengifo. Multivariate autoregressive modelling of time series count data using copulas. Journal of Empirical Finance, 14:564-583, 2007.

[11] Y. Hochberg. A sharper Bonferroni procedure for multiple tests of significance. Biometrika, 75(4):800, 1988. 
[12] Gunnar Högland. Statistical properties of equity transaction data, 2011.

[13] Dorival Leão and Alberto Ohashi. On the discrete cramer-von mises test under censoring. Submetido, 2012.

[14] Philip E. Protter. Stochastic Integration and Differential Equations. Springer, 2th edition, 2005.

[15] T. H. Rydberg and N. Shephard. Dynamics of trade-by-trade price movements: Decomposition and models. Journal of Financial Econometrics, $1: 2-25,2003$.

[16] S.K. Sarkar and C.K. Chang. The simes method for multiple hypothesis testing with positively dependent test statistics. Journal of the American Statistical Association, 92(440):1601-1608, 1997.

[17] A. N. Shiryaev. Probability. Springer, 2th edition, 1995.

[18] R.J. Simes. An improved bonferroni procedure for multiple tests of significance. Biometrika, 73(3):751-754, 1986.

[19] Qiqing Yu, Rick Chappell, G. Y. C. Wong, Yuting Hsu, and Marcin Mazur. Relationship between the cox, lehmann, weibull, and accelerated lifetime models. Communications in Statistics - Theory and Methods, 37:1458-1470, 2008 . 